\title{
Semiclosed projections and applications
}

\author{
Maximiliano Contino $^{\mathrm{a}, \mathrm{b}, *}$, Alejandra Maestripieri ${ }^{\mathrm{a}, \mathrm{b}}$, Stefania Marcantognini ${ }^{\mathrm{b}, \mathrm{c}}$ \\ ${ }^{a}$ Facultad de Ingeniería, Universidad de Buenos Aires \\ Paseo Colón 850 \\ (1063) Buenos Aires, Argentina \\ "Instituto Argentino de Matemática "Alberto P. Calderón" \\ CONICET \\ Saavedra 15, Piso 3 \\ (1083) Buenos Aires, Argentina \\ ${ }^{c}$ Universidad Nacional de General Sarmiento - Instituto de Ciencias \\ Juan María Gutierrez \\ (1613) Los Polvorines, Pcia. de Buenos Aires, Argentina
}

\begin{abstract}
We characterize the semiclosed projections and apply them to compute the Schur complement of a selfadjoint operator with respect to a closed subspace. These projections occur naturally when dealing with weak complementability.
\end{abstract}

Keywords: semiclosed idempotents, operator ranges, complementability

2000 MSC: 47C05, 47A05, 47A64

\section{Introduction}

A linear subspace $\mathcal{E}$ of a Hilbert space $(\mathcal{H},\langle\cdot, \cdot\rangle)$ is semiclosed if there exists an inner product $\langle\cdot, \cdot\rangle^{\prime}$ such that $\left(\mathcal{E},\langle\cdot, \cdot\rangle^{\prime}\right)$ is complete and continuously included in $\mathcal{H}$. The notion of semiclosed subspace was introduced by Kaufman but subspaces of this type appear in the literature before his seminal paper [29]. For instance, in the form of contractively included subspaces in the theory of the de Branges-Rovnyak spaces [18, 19], as the operator ranges of Fillmore and Williams 23] and, under the name of para-closed subspaces, in the work of Foias on the lattice of invariant subspaces 24]. Amongst the semiclosed subspaces of $\mathcal{H} \times \mathcal{H}$, Kaufman pays much attention to those that are graphs of linear operators in $\mathcal{H}$, the so-called semiclosed operators. In fact, the family of all such operators is the main object of analysis in his account of semiclosed subspaces and operators. In the light of Kaufman's study on semiclosed operators we recognize a semiclosed operator $V$ in the factorization $A=B V$ given by the earlier Douglas' Lemma for closed densely defined operators $A, B$ satisfying the operator range inclusion $\mathcal{R}(A) \subseteq \mathcal{R}(B)$, as well as in the Banach space version of the concept of semiclosed operator in a previous work by Caradus [12].

An operator $E$ with domain $\mathcal{D}(E)$ and range $\mathcal{R}(E)$ in $\mathcal{H}$ is a projection provided $\mathcal{R}(E) \subseteq \mathcal{D}(E)$ and $E^{2} x=E x$ for all $x \in \mathcal{D}(E)$. A semiclosed projection $E$ densely defined in a Hilbert space $\mathcal{H}$ occurs when we are given a closed subspace $\mathcal{S}$ of $\mathcal{H}$ and a selfadjoint operator $B$ everywhere defined on $\mathcal{H}$ such that $B$ is $\mathcal{S}$-weakly complementable. Indeed, in this case the Schur complement $B / \mathcal{S}$ of $B$ to $\mathcal{S}$ exists and $B_{/ \mathcal{S}}=(I-E) B$ for some (as a matter of fact, any) semiclosed densely defined projection $E$ in an appropriate class. This fact (cf. [14]) drew our attention to study Hilbert space projections that are densely defined and semiclosed.

Closed projections were studied by Ôta in [35], where he showed that a projection is closed if and only if its nullspace and range are both closed subspaces. We generalize the result to semiclosed projections obtaining the corresponding assertion. Further in-depth investigations on closed projections were carried on by Ando [4]. He proved that a closed densely defined projection $E$ with nullspace $\mathcal{N}$ and range

\footnotetext{
${ }^{*}$ Corresponding author

Email addresses: mcontino@fi.uba.ar (Maximiliano Contino), amaestri@fi.uba.ar (Alejandra Maestripieri), smarcantognini@ungs.edu.ar (Stefania Marcantognini)
} 
$\mathcal{M}$ is distinctively represented as $E=\left(\Gamma^{-1} P_{\mathcal{M}}\right)^{*} \Gamma^{-1}$ where $\Gamma:=\left(P_{\mathcal{M}}+P_{\mathcal{N}}\right)^{1 / 2}$ with $P_{\mathcal{M}}$ and $P_{\mathcal{N}}$ the orthogonal projectors onto $\mathcal{M}$ and $\mathcal{N}$, respectively. Moreover, he established that the well defined operator $\Gamma^{-1} E \Gamma$ is a bounded orthogonal projection. We obtain the analogous result for semiclosed projections with $P_{\mathcal{M}}$ and $P_{\mathcal{N}}$ replaced by $A_{1}$ and $A_{2}$, respectively, where $\left(A_{1}, A_{2}\right)$ is any pair of positive semidefinite operators such that $\mathcal{M}=\mathcal{R}\left(A_{1}\right)$ and $\mathcal{N}=\mathcal{R}\left(A_{2}\right)$. However, in this case, it is not possible to obtain a "distinguished" representation.

Ando also gave a $2 \times 2$ block matrix representation of a closed densely defined projection. The analog for a semiclosed densely defined projection $E$ can be obtained under some extra condition on $\mathcal{D}(E)$. The very same condition allows us to define the Moore-Penrose pseudoinverse $E^{\dagger}$. The case when $E$ is closed was considered in [16]. Therein it was shown that the inverse gives a bijective correspondence between the products of pairs of orthogonal projections and the set of closed densely defined projections. More generally, we prove that the Moore-Penrose pseudoinverse of a semiclosed projection can be related to an operator which is the product of an orthogonal projection times a positive operator. The set of products $P A$, with $P$ an orthogonal projection and $A$ a positive semidefinite operator was studied in [7].

The semiclosed projections are fundamental when studying weak complementability, a concept introduced in [6] for operators in Hilbert spaces which is a generalization of the notion of complementability, introduced by Ando for matrices [2]. The semiclosed projections arisen in this context, when given a selfadjoint operator $B$ on $\mathcal{H}$ and a closed subspace $\mathcal{S}$ of $\mathcal{H}$ such that $B$ is $\mathcal{S}$-weakly complementable, are studied and fully characterized. On the other hand, we study the set of quasi-complementable pairs $(B, \mathcal{S})$, i.e., the set of $B$-symmetric closed projections onto a prescribed subspace $\mathcal{S}$. The relation between the notions of weak complementability and quasi-complementability is analyzed to establish whether they are comparable and to what extent.

Finally, we give a formula of the Schur complement $B / \mathcal{S}$ of a selfadjoint operator $B$ to $\mathcal{S}$ in terms of semiclosed projections. Also, we characterize $B / \mathcal{S}$ as the maximum of a set, when a generalization of the minus order is considered, using again semiclosed projections, see [ [6] and [8].

The paper has five sections including this one. Section 2 is a brief expository introduction to semiclosed subspaces and operators, and serves to set the notation and give some other results that are needed in the following sections. Section 3 is entirely devoted to the study of the class of semiclosed densely defined projections. We also deal with the semiclosed densely defined projections having Moore-Penrose inverses, in particular, those with closed nullspaces. In Section 4 we are concerned with $B$-symmetric projections while in Section 5 we study the notions of weak and quasi complementability and give some applications.

\section{Preliminaries}

We assume that all Hilbert spaces are complex and separable. If $\mathcal{H}$ and $\mathcal{K}$ are Hilbert spaces, by an operator from $\mathcal{H}$ to $\mathcal{K}$ we mean a linear function from a subspace of $\mathcal{H}$ to $\mathcal{K}$. The domain, range, nullspace and graph of any given operator $A$ are denoted by $\mathcal{D}(A), \mathcal{R}(A), \mathcal{N}(A)$ and $\operatorname{gr}(A)$, respectively. Given a subset $\mathcal{T} \subseteq \mathcal{K}$, the preimage of $\mathcal{T}$ under $A$ is $A^{-1}(\mathcal{T}):=\{x \in \mathcal{H}: A x \in \mathcal{T}\} . L(\mathcal{H}, \mathcal{K})$ stands for the space of the bounded linear operators everywhere defined on $\mathcal{H}$ to $\mathcal{K}$. When $\mathcal{H}=\mathcal{K}$ we write, for short, $L(\mathcal{H})$.

The direct sum of two subspaces $\mathcal{M}$ and $\mathcal{N}$ of $\mathcal{H}$ is represented by $\mathcal{M} \dot{+} \mathcal{N}$. If, moreover, $\mathcal{M} \perp \mathcal{N}$ their orthogonal sum is denoted by $\mathcal{M} \oplus \mathcal{N}$. The symbol $\mathcal{Q}$ indicates the subset of the oblique projections in $L(\mathcal{H})$, namely, $\mathcal{Q}:=\left\{Q \in L(\mathcal{H}): Q^{2}=Q\right\}$ and $\mathcal{P}$ the subset of all the orthogonal projections in $L(\mathcal{H})$, $\mathcal{P}:=\left\{P \in L(\mathcal{H}): P^{2}=P=P^{*}\right\}$; for a closed subspace $\mathcal{M}, P_{\mathcal{M}}$ denotes the element in $\mathcal{P}$ with range $\mathcal{M}$.

Denote by $L(\mathcal{H})^{s}$ the set of selfadjoint operators in $L(\mathcal{H}), G L(\mathcal{H})$ the group of invertible operators in $L(\mathcal{H}), L(\mathcal{H})^{+}$the cone of positive semidefinite operators in $L(\mathcal{H})$ and set $G L(\mathcal{H})^{+}:=G L(\mathcal{H}) \cap L(\mathcal{H})^{+}$. Given two operators $S, T \in L(\mathcal{H})$, the notation $T \leq S$ signifies that $S-T \in L(\mathcal{H})^{+}$. Given any $T \in L(\mathcal{H})$, $|T|:=\left(T^{*} T\right)^{1 / 2}$ is the modulus of $T$ and $T=U|T|$ is the polar decomposition of $T$, with $U$ the partial isometry such that $\mathcal{N}(U)=\mathcal{N}(T)$. If $T \in L(\mathcal{H})^{s}$ then $T$ has a unique polar decomposition $T=U|T|$ such that $U=U^{*}=U^{-1}$ and, moreover, $U$ and $|T|$ commute.

Given $B \in L(\mathcal{H})^{s}$ and a (non necessarily closed) subspace $\mathcal{S}$ of $\mathcal{H}$, the $B$-orthogonal complement of $\mathcal{S}$ is $\mathcal{S}^{\perp_{B}}:=\{x \in \mathcal{H}:\langle B x, y\rangle=0$, for every $y \in \mathcal{S}\}=B^{-1}\left(\mathcal{S}^{\perp}\right)=(B \mathcal{S})^{\perp}$.

The next result, due to Fillmore and Williams, characterizes the sum and the intersection of operator ranges as operator ranges. 
Theorem 2.1 (23, Theorem 2.2, Corollary 2]). Let $A, B \in L(\mathcal{H})$. Then

1. $\mathcal{R}(A)+\mathcal{R}(B)=\mathcal{R}\left(\left(A A^{*}+B B^{*}\right)^{1 / 2}\right)$.

2. There exist $X, Y \in L(\mathcal{H})$ such that $\mathcal{R}(A) \cap \mathcal{R}(B)=\mathcal{R}\left(\left(A X A^{*}\right)^{1 / 2}\right)=\mathcal{R}\left(\left(B Y B^{*}\right)^{1 / 2}\right)$.

Given $B \in L(\mathcal{H})^{s}$ and $\mathcal{S}$ a closed subspace of $\mathcal{H}$, we say that $\mathcal{S}$ is $B$-positive if $\langle B s, s\rangle>0$ for every $s \in$ $\mathcal{S}, s \neq 0$. B-nonnegative, B-neutral, B-negative and B-nonpositive subspaces are defined analogously. If $\mathcal{S}$ and $\mathcal{T}$ are two closed subspaces of $\mathcal{H}$, the notation $\mathcal{S} \oplus_{B} \mathcal{T}$ is used to indicate the direct sum of $\mathcal{S}$ and $\mathcal{T}$ when, in addition, $\langle B s, t\rangle=0$ for every $s \in \mathcal{S}$ and $t \in \mathcal{T}$.

The following is a consequence of the spectral theorem for Hilbert space selfadjoint operators.

Lemma 2.2. Let $B \in L(\mathcal{H})^{s}$ and $\mathcal{S}$ be a closed subspace of $\mathcal{H}$. Then the Grammian of $B, G_{B, \mathcal{S}}:=\left.P_{\mathcal{S}} B\right|_{\mathcal{S}}$ can be represented as

$$
G_{B, \mathcal{S}}=G_{1}-G_{2},
$$

where $G_{1}, G_{2} \in L(\mathcal{S})^{+}$and $\mathcal{R}\left(G_{1}\right) \perp \mathcal{R}\left(G_{2}\right)$. Also, if $\mathcal{S}_{+}:=\overline{\mathcal{R}\left(G_{1}\right)}$ and $\mathcal{S}_{-}:=\overline{\mathcal{R}\left(G_{2}\right)} \oplus \mathcal{N}\left(G_{B, \mathcal{S}}\right)$, then $\mathcal{S}_{+} \perp \mathcal{S}_{-}$and $\mathcal{S}$ can be represented as

$$
\mathcal{S}=\mathcal{S}_{+} \oplus_{B} \mathcal{S}_{-},
$$

where $\mathcal{S}_{+}$is B-positive and $\mathcal{S}_{-}$is B-nonpositive.

Let $A, B \in L(\mathcal{H})$. Then, by Douglas' Lemma [22], $\mathcal{R}(B) \subseteq \mathcal{R}(A)$ if and only the equation $A X=B$ has a solution in $L(\mathcal{H})$. In this case, there exists a unique $D$ such that $A D=B$ and $\mathcal{R}(D) \subseteq \overline{\mathcal{R}\left(A^{*}\right)}$. The operator $D$ is called the reduced solution of the equation $A X=B$.

The next lemma characterizes the positive operators in terms of its matrix decomposition, see [1].

Lemma 2.3. Let $\mathcal{S} \subseteq \mathcal{H}$ be a closed subspace and $B \in L(\mathcal{H})^{s}$ with matrix decomposition

$$
B=\left[\begin{array}{cc}
a & b \\
b^{*} & c
\end{array}\right] \begin{gathered}
\mathcal{S} \\
\mathcal{S}^{\perp}
\end{gathered}
$$

Then $B \in L(\mathcal{H})^{+}$if and only if

$$
a \geq 0, \mathcal{R}(b) \subseteq \mathcal{R}\left(a^{1 / 2}\right) \text { and } c=f^{*} f+t,
$$

for some $t \geq 0$, where $f$ is the reduced solution of the equation $b=a^{1 / 2} x$.

\section{Semiclosed subspaces and operators}

The notions of semiclosed subspace and semiclosed operator were formally introduced by Kaufman [29], though these notions were considered by other authors before, as we pointed out in the Introduction.

Definition. A subspace $\mathcal{S}$ of $\mathcal{H}$ is semiclosed if $\mathcal{S}$ is a (not necessarily closed) subspace for which there exists an inner product $\langle\cdot, \cdot\rangle^{\prime}$ such that $\left(\mathcal{S},\langle\cdot, \cdot\rangle^{\prime}\right)$ is a Hilbert space which is continuosly included in $\mathcal{H}$, i.e., there exists $b>0$ such that $\langle x, x\rangle \leq b\langle x, x\rangle^{\prime}$ for every $x \in \mathcal{S}$.

As only an infinite dimensional subspace can be semiclosed but not closed, only infinite dimensional complex Hilbert spaces are considered.

Operator ranges are semiclosed subspaces: in fact, if $T \in L(\mathcal{H})$ define

$$
\|u\|_{T}:=\left\|T^{\dagger} u\right\| \text { for } u \in \mathcal{R}(T),
$$

where $T^{\dagger}$ denotes the (possibly unbounded) Moore-Penrose inverse of $T$, see [34]. Then $\left(\mathcal{R}(T),\|\cdot\|_{T}\right)$ is a Hilbert space and

$$
\|u\|=\left\|T T^{\dagger} u\right\| \leq\|T\|\left\|T^{\dagger} u\right\|=\|T\|\|u\|_{T} \text { for } u \in \mathcal{R}(T) .
$$

See [3, 11].

The space $\mathcal{R}(T)$ equipped with the Hilbert space structure $\|\cdot\|_{T}$ is denoted by

$$
\mathcal{M}(T):=\left(\mathcal{R}(T),\|\cdot\|_{T}\right) .
$$


The Hilbert spaces $\mathcal{M}(T)$ play a significant role in many areas, in particular in the de Branges complementation theory [3].

The semiclosed subspaces are all of them operator ranges: Fillmore and Williams proved that $\mathcal{S}$ is a semiclosed subspace of $\mathcal{H}$ if and only if $\mathcal{S}$ is the range of a closed operator $T$ on $\mathcal{H}$. Moreover, the operator $T$ can be chosen to be bounded and positive (semidefinite), see 23, Theorem 1.1]. Furthermore, if $T$ is a contraction, i.e. $\|T\| \leq 1$, then $\mathcal{S}^{\prime}:=\mathcal{M}\left(\left(I-T T^{*}\right)^{1 / 2}\right)$ is its de Branges complement and $\mathcal{S}+\mathcal{S}^{\prime}=\mathcal{H}$ [3, Corollary 3.8], where the last sum need not be direct [11, Proposition 3.4].

Given two operators $T_{1}, T_{2} \in L(\mathcal{H})$, by Theorem 2.1] the subspace $\mathcal{R}\left(T_{1}\right)+\mathcal{R}\left(T_{2}\right)$ is the range of $T:=\left(T_{1} T_{1}^{*}+T_{2} T_{2}^{*}\right)^{1 / 2}$. This shows that the sum of semiclosed subspaces is again semiclosed. The following interesting result by Ando compares the norm $\|\cdot\|_{T}$ with the norms $\|\cdot\|_{T_{1}}$ and $\|\cdot\|_{T_{2}}$.

Theorem 2.4 ([3, Corollary 3.8]). For $T_{1}, T_{2} \in L(\mathcal{H})$, let $T:=\left(T_{1} T_{1}^{*}+T_{2} T_{2}^{*}\right)^{1 / 2}$. Then $\left\|u_{1}+u_{2}\right\|_{T}^{2} \leq$ $\left\|u_{1}\right\|_{T_{1}}^{2}+\left\|u_{2}\right\|_{T_{2}}^{2}$, for $u_{1} \in \mathcal{R}\left(T_{1}\right)$ and $u_{2} \in \mathcal{R}\left(T_{2}\right)$, and for any $u \in \mathcal{R}(T)$, there are unique $u_{1} \in \mathcal{R}\left(T_{1}\right)$ and $u_{2} \in \mathcal{R}\left(T_{2}\right)$ such that $u=u_{1}+u_{2}$ and

$$
\left\|u_{1}+u_{2}\right\|_{T}^{2}=\left\|u_{1}\right\|_{T_{1}}^{2}+\left\|u_{2}\right\|_{T_{2}}^{2} .
$$

Applying again Theorem 2.1 it follows that the family of semiclosed subspaces is closed under intersection, see also [10, Proposition 4, Proposition 6]. The set of semiclosed subspaces is the lattice of domains of closed operators in $\mathcal{H}[23]$. Also, if $\mathcal{S}$ is a semiclosed subspace of $\mathcal{H}$ then all inner products $\langle\cdot, \cdot\rangle^{\prime}$ such that $\left(\mathcal{S},\langle\cdot, \cdot\rangle^{\prime}\right)$ is a Hilbert space which is continuosly included in $\mathcal{H}$ generate the same topology on $\mathcal{S}$. See [31, 32] and [10, Theorem 11].

Definition $(29,30])$. An operator $C: \mathcal{D}(C) \subseteq \mathcal{H} \rightarrow \mathcal{K}$ is a semiclosed operator if $\operatorname{gr}(C)$ is a semiclosed subspace of $\mathcal{H} \times \mathcal{K}$.

Denote by $S C(\mathcal{H}, \mathcal{K})$ the set of all semiclosed operators with domain in $\mathcal{H}$ to $\mathcal{K}$ and $\operatorname{set} S C(\mathcal{H}):=$ $S C(\mathcal{H}, \mathcal{H})$. The following is a characterization of $S C(\mathcal{H})$, see $[29$, Theorem 1].

Theorem 2.5. Given an operator $C: \mathcal{D}(C) \subseteq \mathcal{H} \rightarrow \mathcal{H}$, the following are equivalent:

i) $C \in S C(\mathcal{H})$;

ii) $\mathcal{D}(C)$ is a semiclosed subspace of $\mathcal{H}$ and $C \in L(\mathcal{D}(C), \mathcal{H})$;

iii) there exist $A \in L(\mathcal{H})$ and $D \in L(\mathcal{H})^{+}$such that $C=\left.A D^{\dagger}\right|_{\mathcal{R}(D)}$ and $\mathcal{N}(D) \subseteq \mathcal{N}(A)$.

Corollary 2.6. Let $C: \mathcal{D}(C) \subseteq \mathcal{H} \rightarrow \mathcal{H}$ be a given operator. Then $C \in S C(\mathcal{H})$ if and only if there exists $D \in L(\mathcal{H})^{+}$such that $\mathcal{D}(C)=\mathcal{R}(D)$ and $C D \in L(\mathcal{H})$.

Corollary 2.7. Let $C \in S C(\mathcal{H})$. Then $C A \in L(\mathcal{K}, \mathcal{H})$ for any $A \in L(\mathcal{K}, \mathcal{H})$ such that $\mathcal{R}(A) \subseteq \mathcal{D}(C)$.

Proof. By Corollary 2.6 there exists an operator $D \in L(\mathcal{H})^{+}$such that $\mathcal{D}(C)=\mathcal{R}(D)$ and $C D \in L(\mathcal{H})$. Then, if $\mathcal{R}(A) \subseteq \mathcal{D}(C)=\mathcal{R}(D)$, by Douglas' Lemma, $A=D X_{0}$ for some $X_{0} \in L(\mathcal{K}, \mathcal{H})$. Therefore, $C A=C D X_{0} \in L(\mathcal{K}, \mathcal{H})$.

The set $S C(\mathcal{H})$ is closed under addition, multiplication, inversion and restriction to semiclosed subspaces of $\mathcal{H}$ [29]. Also, if $T_{1}, T_{2} \in S C(\mathcal{H}, \mathcal{K})$ are such that $T_{1}$ and $T_{2}$ coincide on $\mathcal{D}\left(T_{1}\right) \cap \mathcal{D}\left(T_{2}\right)$, then the operator $T: \mathcal{D}\left(T_{1}\right)+\mathcal{D}\left(T_{2}\right) \rightarrow \mathcal{K}$ coinciding with $T_{1}$ on $\mathcal{D}\left(T_{1}\right)$ and with $T_{2}$ on $\mathcal{D}\left(T_{2}\right)$ is a semiclosed operator 21].

Remark. In 22, Theorem 2], Douglas proved that given $A, B$ densely defined closed operators on $\mathcal{H}$ such that $\mathcal{R}(A) \subseteq \mathcal{R}(B)$, there exist an operator $V$ on $\mathcal{H}$ with $\mathcal{D}(V)=\mathcal{D}(A)$ and a number $M \geq 0$ such that

$$
A=B V \text { and }\|V x\|^{2} \leq M\left(\|x\|^{2}+\|A x\|^{2}\right), \text { for every } x \in \mathcal{D}(V) .
$$

The operator $V$ is semiclosed: in fact, define

$$
\langle(x, V x),(y, V y)\rangle^{\prime}:=\langle x, y\rangle+\langle A x, A y\rangle \text { for } x, y \in \mathcal{D}(V) .
$$

Then $\left(\operatorname{gr}(V),\langle\cdot, \cdot\rangle^{\prime}\right)$ is a Hilbert space because $A$ is closed. On the other hand, since (2.4) holds,

$$
\langle(x, V x),(x, V x)\rangle \leq(M+1)\langle(x, V x),(x, V x)\rangle^{\prime} \text { for every } x \in \mathcal{D}(V) .
$$

Hence $\left(\operatorname{gr}(V),\langle\cdot, \cdot\rangle^{\prime}\right)$ is continuously included in $\mathcal{H} \times \mathcal{H}$. 


\section{Semiclosed projections}

A linear operator $E$ acting in $\mathcal{H}$ is a projection if

$$
\mathcal{R}(E) \subseteq \mathcal{D}(E) \text { and } E^{2} x=E x \text { for every } x \in \mathcal{D}(E) .
$$

Theorem 3.1 ([35, Lemma 3.5]). 1. If $E$ is a projection in $\mathcal{H}$ then

$$
\mathcal{R}(E) \dot{+} \mathcal{N}(E)=\mathcal{D}(E) .
$$

Conversely, given two subspaces $\mathcal{N}, \mathcal{M}$ of $\mathcal{H}$ such that $\mathcal{N} \cap \mathcal{M}=\{0\}$, there exists a projection $E$ with $\mathcal{R}(E)=\mathcal{M}$ and $\mathcal{N}(E)=\mathcal{N}$.

2. $E$ is a closed projection if and only if $\mathcal{R}(E)$ and $\mathcal{N}(E)$ are closed subspaces of $\mathcal{H}$.

Write $E=P_{\mathcal{M} / \mathcal{N}}$ to denote the projection with $\mathcal{R}(E)=\mathcal{M}$ and $\mathcal{N}(E)=\mathcal{N}$. If $E$ is a densely defined projection in $\mathcal{H}$, then $E^{*}$ is a (non necessarily densely defined) closed projection (see 35, Proposition 3.4]) with $\mathcal{N}\left(E^{*}\right)=\mathcal{R}(E)^{\perp}$ and $\mathcal{R}\left(E^{*}\right)=\mathcal{N}(E)^{\perp}$. The last equality follows from the former and the fact that $I-E$ is a projection with domain $\mathcal{D}(E)$ so that $\mathcal{R}\left(E^{*}\right)=\mathcal{N}\left(I-E^{*}\right)=\mathcal{R}(I-E)^{\perp}=\mathcal{N}(E)^{\perp}$. Then, by Theorem 3.1

$$
\mathcal{D}\left(E^{*}\right)=\mathcal{N}(E)^{\perp} \dot{+} \mathcal{R}(E)^{\perp} .
$$

From this, it is immediate that if $E$ is a densely defined closed projection, then $E^{*}$ is densely defined.

Every densely defined closed projection $E=P_{\mathcal{M} / \mathbb{N}}$ admits a matrix representation according to the decomposition $\mathcal{H}=\mathcal{M} \oplus \mathcal{M}^{\perp}$,

$$
E=\left[\begin{array}{ll}
1 & x \\
0 & 0
\end{array}\right]
$$

such that $x: \mathcal{D}(x) \subseteq \mathcal{M}^{\perp} \rightarrow \mathcal{M}$ is a densely defined closed operator, where $\mathcal{D}(x)=P_{\mathcal{M}^{\perp}}(\mathcal{N})$.

Conversely, consider the decomposition $\mathcal{H}=\mathcal{M} \oplus \mathcal{M}^{\perp}$. If

$$
E=\left[\begin{array}{ll}
1 & x \\
0 & 0
\end{array}\right]
$$

where $x: \mathcal{D}(x) \subseteq \mathcal{M}^{\perp} \rightarrow \mathcal{M}$ is a densely defined operator and $\mathcal{D}(E)=\mathcal{M} \oplus \mathcal{D}(x)$. Then $E$ is a densely defined projection with $\mathcal{R}(E)=\mathcal{M}$. Furthermore, $E$ is closed if and only if $x$ is closed. In this case,

$$
E^{*}=\left[\begin{array}{cc}
1 & 0 \\
x^{*} & 0
\end{array}\right]
$$

where $x^{*}: \mathcal{D}\left(x^{*}\right) \subseteq \mathcal{M} \rightarrow \mathcal{M}^{\perp}$ is densely defined. See [15, Proposition 1.7] for the proof of these assertions.

More generally,

Proposition 3.2. Let $E: \mathcal{D}(E) \subseteq \mathcal{H} \rightarrow \mathcal{H}$ be a projection. Then $E$ is semiclosed if and only if $\mathcal{R}(E)$ and $\mathcal{N}(E)$ are semiclosed subspaces.

Proof. If $\mathcal{R}(E)$ and $\mathcal{N}(E)$ are semiclosed subspaces of $\mathcal{H}$, there exist $A_{1}, A_{2} \in L(\mathcal{H})^{+}$such that $\mathcal{R}(E)=$ $\mathcal{R}\left(A_{1}\right)$ and $\mathcal{N}(E)=\mathcal{R}\left(A_{2}\right)$. Then, by Theorem 2.1. $\mathcal{D}(E)=\mathcal{R}(E) \dot{+} \mathcal{N}(E)$ is semiclosed. Let us see that $E \in L(\mathcal{D}(E), \mathcal{H})$. Consider $\Gamma=\left(A_{1}^{2}+A_{2}^{2}\right)^{1 / 2}$. Then, by Theorem 2.1, $\mathcal{R}(\Gamma)=\mathcal{R}\left(A_{1}\right)+\mathcal{R}\left(A_{2}\right)=\mathcal{D}(E)$. Let $u \in \mathcal{D}(E)$. Then, by Theorem 2.4 there exist uniquely $m \in \mathcal{R}(E)$ and $n \in \mathcal{N}(E)$ such that $u=m+n$ and $\|u\|_{\Gamma}^{2}=\|m\|_{A_{1}}^{2}+\|n\|_{A_{2}}^{2}$. Then, using (2.3),

$$
\|E u\|^{2}=\|m\|^{2} \leq\left\|A_{1}\right\|^{2}\|m\|_{A_{1}}^{2} \leq\left\|A_{1}\right\|^{2}\left(\|m\|_{A_{1}}^{2}+\|n\|_{A_{2}}^{2}\right)=\left\|A_{1}\right\|^{2}\|u\|_{\Gamma}^{2} .
$$

Then $E \in L(\mathcal{D}(E), \mathcal{H})$ and, by Theorem 2.5, $E \in S C(\mathcal{H})$.

Conversely, suppose that $E$ is a semiclosed projection. Then, by Theorem 2.5, $\mathcal{D}(E)$ is a semiclosed subspace of $\mathcal{H}$ and, by [29, Theorem 2], $\mathcal{R}(E)=E(\mathcal{D}(E))$ is also a semiclosed subspace of $\mathcal{H}$. Since the set $S C(\mathcal{H})$ is closed under addition, $E \in S C(\mathcal{H})$ if and only if $I-E \in S C(\mathcal{H})$. Hence, $\mathcal{N}(E)=\mathcal{R}(I-E)$ is a semiclosed subspace of $\mathcal{H}$, where we used again [29, Theorem 2]. 
Definition. Let

$\mathcal{Q}_{S C}:=\{E: E$ is a densely defined semiclosed projection on $\mathcal{H}\}$

In [4, Theorem 2.2], Ando proved that if $E=P_{\mathcal{M} / \mathcal{N}}$ is a closed projection and $\Gamma:=\left(P_{\mathcal{M}}+P_{\mathcal{N}}\right)^{1 / 2}$ then $\mathcal{D}(E)=\mathcal{R}(\Gamma)$ and $E$ admits the following representation:

$$
E=\left(\Gamma^{-1} P_{\mathcal{M}}\right)^{*} \Gamma^{-1} .
$$

Moreover, the well defined operator $P:=\Gamma^{-1} E \Gamma$ is an orthogonal projection, see [4, Theorem 2.3]. Analogous results can be obtained for densely defined semiclosed projections.

Let $E=P_{\mathcal{M} / \mathcal{N}} \in \mathcal{Q}_{S C}$. Since, by Proposition 3.2, $\mathcal{M}$ and $\mathcal{N}$ are semiclosed subspaces, there exist $A_{1}, A_{2} \in L(\mathcal{H})^{+}$such that $\mathcal{M}=\mathcal{R}\left(A_{1}\right)$ and $\mathcal{N}=\mathcal{R}\left(A_{2}\right)$. Define the operator $\Gamma=\Gamma\left(A_{1}, A_{2}\right)$ as

$$
\Gamma:=\left(A_{1}^{2}+A_{2}^{2}\right)^{1 / 2} \text {. }
$$

Then $\Gamma \in L(\mathcal{H})^{+}$and, by Theorem 2.1, $\mathcal{R}(\Gamma)=\mathcal{R}\left(A_{1}\right) \dot{+} \mathcal{R}\left(A_{2}\right)=\mathcal{D}(E)$ is dense, so that $\Gamma$ is injective.

Proposition 3.3 (cf. [4, Theorem 2.2]). Let $E \in \mathcal{Q}_{S C}$ with $\mathcal{R}(E)=\mathcal{R}\left(A_{1}\right), \mathcal{N}(E)=\mathcal{R}\left(A_{2}\right), A_{1}, A_{2} \in$ $L(\mathcal{H})^{+}$and $\Gamma$ as in (3.4). Then $E$ admits the representation

$$
E=\left(\Gamma^{-1} A_{1}^{2}\right)^{*} \Gamma^{-1}
$$

Proof. Since $\mathcal{R}\left(A_{1}^{2}\right) \subseteq \mathcal{R}\left(A_{1}\right) \subseteq \mathcal{R}(\Gamma)$ and $\Gamma$ is injective, by Douglas' Lemma, there exists a unique $D \in$ $L(\mathcal{H})$ such that $A_{1}^{2}=\Gamma D^{*}=D \Gamma$, then $D^{*}=\Gamma^{-1} A_{1}^{2}$ and $D=\left(\Gamma^{-1} A_{1}^{2}\right)^{*}$. Write $\tilde{E}=D \Gamma^{-1}$. Since $\mathcal{D}(E)=$ $\mathcal{R}(\Gamma)=\mathcal{D}(\tilde{E})$, for the proof of the assertion it suffices to show that $E \Gamma x=\tilde{E} \Gamma x=D x$ for every $x \in \mathcal{H}$. Since $E \Gamma$ is bounded by Corollary 2.7, and $\mathcal{R}(\Gamma)$ is dense in $\mathcal{H}$, the equality is guaranteed if the operators $E \Gamma$ and $D$ coincide on this dense subspace. It is $E \Gamma^{2} x=E\left(A_{1}^{2}+A_{2}^{2}\right) x=A_{1}^{2} x=D \Gamma x$. Therefore $E \Gamma=D$ or $E=\tilde{E}=D \Gamma^{-1}$ on $\mathcal{R}(\Gamma)$.

Corollary 3.4 (cf. [4, Theorem 2.3]). Let $E$ be a densely defined operator in $\mathcal{H}$. Then $E \in \mathcal{Q}_{S C}$ if and only if there exists $\Gamma \in L(\mathcal{H})^{+}$injective with $\mathcal{R}(\Gamma)=\mathcal{D}(E)$ such that

$$
\Gamma^{-1} E \Gamma \in \mathcal{P} \text {. }
$$

Proof. Suppose that $E \in \mathcal{Q}_{S C}$ with $\mathcal{R}(E)=\mathcal{R}\left(A_{1}\right), \mathcal{N}(E)=\mathcal{R}\left(A_{2}\right), A_{1}, A_{2} \in L(\mathcal{H})^{+}$. Let $D:=$ $\left(\Gamma^{-1} A_{1}^{2}\right)^{*}$, with $\Gamma$ as in (3.4). Then $\Gamma$ is injective and, by Proposition 3.3. $E \Gamma=D$. Let $P_{\Gamma}:=\Gamma^{-1} E \Gamma=$ $\Gamma^{-1} D$, then $P_{\Gamma}$ is a bounded projection. In fact, since $\mathcal{R}(D) \subseteq \mathcal{R}(E) \subseteq \mathcal{D}(E)=\mathcal{R}(\Gamma)$, by Douglas' Lemma, the only solution $X_{0}$ of the equation $D=\Gamma X$ is given by $X_{0}=\Gamma^{-1} D \in L(\mathcal{H})$. Also, since $\mathcal{R}\left(A_{1}\right) \subseteq \mathcal{R}(\Gamma)$, by Douglas's Lemma again, there exists a unique $D^{\prime} \in L(\mathcal{H})$ such that $A_{1}=\Gamma D^{\prime *}=D^{\prime} \Gamma$, then $D^{\prime *}=\Gamma^{-1} A_{1}$ and $D^{\prime}=\left(\Gamma^{-1} A_{1}\right)^{*}$. From $\Gamma\left(D^{\prime *} \Gamma D^{\prime *}\right)=A_{1}^{2}=\Gamma D^{*}$ and the fact that $\Gamma$ is injective, it follows that $D=D^{\prime} \Gamma D^{\prime}$. Then $P_{\Gamma}=\Gamma^{-1} D=\Gamma^{-1} D^{\prime} \Gamma D^{\prime}=\Gamma^{-1} A_{1} D^{\prime}=\left(D^{\prime}\right)^{*} D^{\prime} \in L(\mathcal{H})^{s}$. Also $P_{\Gamma}^{2}=\Gamma^{-1} D \Gamma^{-1} D=\Gamma^{-1} E \Gamma \Gamma^{-1} D=\Gamma^{-1} E D=\Gamma^{-1} D=P_{\Gamma}$.

Conversely, suppose that $E$ is a densely defined operator in $\mathcal{H}$ such that there exists $\Gamma \in L(\mathcal{H})^{+}$with $\mathcal{R}(\Gamma)=\mathcal{D}(E)$ and $\Gamma^{-1} E \Gamma:=P_{\Gamma} \in \mathcal{P}$. Then $E \Gamma=\Gamma P_{\Gamma}$ and, by Corollary 2.6. $E \in S C(\mathcal{H})$. Since $\Gamma$ is injective, $E=\Gamma P_{\Gamma} \Gamma^{-1}, \mathcal{R}(E) \subseteq \mathcal{D}(E)$ and $E^{2}=E$.

Since $E \Gamma=\Gamma P_{\Gamma}$, it follows that $P_{\Gamma}=P_{\Gamma^{-1}(\mathcal{R}(E))}$. Since $\mathcal{R}(E)=\Gamma\left(\mathcal{R}\left(P_{\Gamma}\right)\right), \mathcal{N}(E)=\Gamma\left(\mathcal{N}\left(P_{\Gamma}\right)\right)$ and $P_{\Gamma}$ is orthogonal, it holds that $\Gamma^{-1}(\mathcal{R}(E)) \perp \Gamma^{-1}(\mathcal{N}(E))$.

If $\Gamma, \Gamma^{\prime} \in L(\mathcal{H})^{+}$are as in Corollary 3.4 then $\mathcal{R}(\Gamma)=\mathcal{D}(E)=\mathcal{R}\left(\Gamma^{\prime}\right)$ and, by Douglas' Lemma, there exists $G \in G L(\mathcal{H})$ such that $\Gamma^{\prime}=\Gamma G=G^{*} \Gamma$. Moreover, if $P_{\Gamma}=P_{\Gamma^{-1}(\mathcal{R}(E))}$ and $P_{\Gamma^{\prime}}=P_{\Gamma^{\prime-1}}(\mathcal{R}(E))$ then

$$
P_{\Gamma^{\prime}}=G^{-1} P_{\Gamma} G \text {. }
$$

In fact, the projection $G^{-1} P_{\Gamma} G$ is bounded, $\mathcal{R}\left(G^{-1} P_{\Gamma} G\right)=G^{-1}\left(\mathcal{R}\left(P_{\Gamma}\right)\right)=(\Gamma G)^{-1}(\mathcal{R}(E))=\Gamma^{\prime-1}(\mathcal{R}(E))$ $=\mathcal{R}\left(P_{\Gamma^{\prime}}\right)$ and $\mathcal{N}\left(G^{-1} P_{\Gamma} G\right)=G^{-1}\left(\mathcal{N}\left(P_{\Gamma}\right)\right)=(\Gamma G)^{-1}(\mathcal{N}(E))=\Gamma^{\prime-1}(\mathcal{N}(E))=\mathcal{N}\left(P_{\Gamma^{\prime}}\right)$. 


\subsection{On the Moore-Penrose inverse of semiclosed projections with closed nullspace}

In order to define the Moore-Penrose inverse of a densely defined projection $E$ in a satisfactory fashion we need an extra condition on its domain. This condition guarantees the existence of an orthogonal complement of $\mathcal{N}(E)$ relative to $\mathcal{D}(E)$.

Lemma 3.5. Let $E=P_{\mathcal{M} / \mathbb{N}}$ be a densely defined projection. Then the following statements are equivalent:

i) $\mathcal{M} \subseteq \mathcal{N}^{\perp} \oplus \mathcal{N}$

ii) $\mathcal{D}(E)=P_{\mathcal{N}^{\perp}}(\mathcal{M}) \oplus \mathcal{N}$;

iii) $\mathcal{D}(E)=\mathcal{D}(E) \cap \mathcal{N}^{\perp} \oplus \mathcal{N}$.

In this case, $\mathcal{M} \cap \overline{\mathcal{N}}=\{0\}$ and therefore $E$ admits the extension $P_{\mathcal{M} / \overline{\mathcal{N}}}$.

Proof. $\quad$ i) $\Rightarrow$ ii) : Let $x \in \mathcal{M}$. Then $x=P_{\mathcal{N}^{\perp}} x+n$, for some $n \in \mathcal{N}$. Therefore $x \in P_{\mathcal{N}^{\perp}}(\mathcal{M}) \oplus \mathcal{N}$, $\mathcal{M} \subseteq P_{\mathcal{N}^{\perp}}(\mathcal{M}) \oplus \mathcal{N}$ and $\mathcal{D}(E)=\mathcal{M} \dot{+} \mathcal{N} \subseteq P_{\mathcal{N}^{\perp}}(\mathcal{M}) \oplus \mathcal{N}$. To see the other inclusion, if $y \in P_{\mathcal{N}^{\perp}}(\mathcal{M})$ then there exists $m \in \mathcal{M}$ such that $y=P_{\mathcal{N} \perp} m$. Since $m \in \mathcal{M}$, there exists $t \in \mathcal{N}^{\perp}$ and $n \in \mathcal{N}$ such that $m=t+n$. Then $y=m-\left(I-P_{\mathcal{N}^{\perp}}\right) m=m-n \in \mathcal{M} \dot{+} \mathcal{N}$. Then $P_{\mathcal{N}^{\perp}}(\mathcal{M}) \subseteq \mathcal{D}(E)$ and $P_{\mathcal{N}^{\perp}}(\mathcal{M}) \oplus \mathcal{N} \subseteq \mathcal{D}(E)$.

ii) $\Rightarrow$ iii) : Clearly, $P_{\mathcal{N}^{\perp}}(\mathcal{M}) \subseteq \mathcal{D}(E) \cap \mathcal{N}^{\perp}$. On the other hand, let $x \in \mathcal{D}(E) \cap \mathcal{N}^{\perp}$. Then $x=m+n$, for some $m \in \mathcal{M}$ and $n \in \mathcal{N}$ and $x=P_{\mathcal{N}^{\perp}} x=P_{\mathcal{N}^{\perp} m} \in P_{\mathcal{N}^{\perp}}(\mathcal{M})$. Therefore $P_{\mathcal{N}^{\perp}}(\mathcal{M})=\mathcal{D}(E) \cap \mathcal{N}^{\perp}$ and $\mathcal{D}(E)=\mathcal{D}(E) \cap \mathcal{N}^{\perp} \oplus \mathcal{N}$.

iii) $\Rightarrow$ i) : It follows from the fact that $\mathcal{M} \subseteq \mathcal{D}(E)=\mathcal{D}(E) \cap \mathcal{N}^{\perp} \oplus \mathcal{N} \subseteq \mathcal{N}^{\perp} \oplus \mathcal{N}$.

In this case, from $\mathcal{M} \subseteq \mathcal{N} \oplus \mathcal{N}^{\perp}$, we have that $P_{\overline{\mathcal{N}}}(\mathcal{M}) \subseteq \mathcal{N}$. Therefore $\mathcal{M} \cap \overline{\mathcal{N}}=\{0\}$. In fact, if $x \in \mathcal{M} \cap \overline{\mathcal{N}}$. Then $x=P_{\overline{\mathcal{N}}} x \in \mathcal{N} \cap \mathcal{M}=\{0\}$.

Given $E$ a densely defined projection, condition $i)$ on $\mathcal{R}(E)$ and $\mathcal{N}(E)$ need not hold: the example on page 278 of [23] shows that there exist subspaces $\mathcal{M}, \mathcal{N}$ of $\mathcal{H}$ such that $\overline{\mathcal{M} \dot{+} \mathcal{N}}=\mathcal{H}$, but $\mathcal{M} \cap \overline{\mathcal{N}} \neq\{0\}$.

We begin by applying Lemma 3.5 to obtain a matrix decomposition of a given projection. Every closed projection $P_{\mathcal{M} / \mathcal{N}}$ admits a matrix representation according to the orthogonal decomposition $\mathcal{H}=\mathcal{N}^{\perp} \oplus \mathcal{N}$ on $\mathcal{D}(E)=P_{\mathcal{N}^{\perp}}(\mathcal{M}) \oplus \mathcal{N}$, see [4, Theorem 2.6]. We generalize this result for a densely defined projection $E=P_{\mathcal{M} / \mathbb{N}}$ satisfying the conditions of Lemma 3.5

Proposition 3.6 (cf. [4, Theorem 2.6]). Let $E=P_{\mathcal{M} / \mathcal{N}}$ be a densely defined projection such that $\mathcal{M} \subseteq$ $\mathcal{N} \oplus \mathcal{N}^{\perp}$. According to the orthogonal decomposition $\mathcal{H}=\mathcal{N}^{\perp} \oplus \overline{\mathcal{N}}, E$ admits the matrix representation

$$
E=\left[\begin{array}{cc}
I & 0 \\
P_{\overline{\mathcal{N}}}\left(\left.P_{\mathcal{N}^{\perp}} P_{\overline{\mathcal{M}}}\right|_{\mathcal{M}}\right)^{-1} & 0
\end{array}\right] \text { on } P_{\mathcal{N}^{\perp}}(\mathcal{M}) \oplus \mathcal{N} .
$$

Proof. By Lemma 3.5. the operator $\left.P_{\mathcal{N}^{\perp}} P_{\overline{\mathcal{M}}}\right|_{\mathcal{M}}$ is injective with dense range in $\mathcal{N}^{\perp}$. In fact, if $P_{\mathcal{N}^{\perp}} P_{\overline{\mathcal{M}}} x=0$ for $x \in \mathcal{M}$, then $x \in \mathcal{M} \cap \overline{\mathcal{N}}=\{0\}$. Also, $\mathcal{N}^{\perp}=P_{\mathcal{N}^{\perp}}(\overline{\mathcal{M}+\mathcal{N}}) \subseteq \overline{P_{\mathcal{N}^{\perp}}(\mathcal{M})} \subseteq \mathcal{N}^{\perp}$. So that $\overline{\mathcal{R}\left(\left.P_{\mathcal{N}^{\perp}} P_{\overline{\mathcal{M}}}\right|_{\mathcal{M}}\right)}=\overline{P_{\mathcal{N}^{\perp}}(\mathcal{M})}=\mathcal{N}^{\perp}$ and the operator $P_{\overline{\mathcal{N}}}\left(\left.P_{\mathcal{N}^{\perp}} P_{\overline{\mathcal{M}}}\right|_{\mathcal{M}}\right)^{-1}$ is a linear operator from $P_{\mathcal{N}^{\perp}}(\mathcal{M}) \subseteq \mathcal{N}^{\perp}$ to $\mathcal{N}$.

By Lemma 3.5 again, $\mathcal{D}(E)=P_{\mathcal{N}^{\perp}}(\mathcal{M}) \oplus \mathcal{N}$ and $P_{\mathcal{N}^{\perp}}(\mathcal{M})=\mathcal{D}(E) \cap \mathcal{N}^{\perp}$. Clearly, $\left.E P_{\overline{\mathcal{N}}}\right|_{\mathcal{N}}=0$ and $\left.P_{\mathcal{N}^{\perp}} E P_{\mathcal{N}^{\perp}}\right|_{\mathcal{D}(E) \cap \mathcal{N}^{\perp}}=\left.I_{\mathcal{N}^{\perp}}\right|_{\mathcal{D}(E) \cap \mathcal{N}^{\perp}}$. Finally, $\left.P_{\overline{\mathcal{N}}} E P_{\mathcal{N}^{\perp}}\right|_{\mathcal{D}(E) \cap \mathcal{N}^{\perp}}=P_{\overline{\mathcal{N}}}\left(\left.P_{\mathcal{N}^{\perp}} P_{\overline{\mathcal{M}}}\right|_{\mathcal{M}}\right)^{-1}$ on $P_{\mathcal{N}^{\perp}}(\mathcal{M})$.

Let $E=P_{\mathcal{M} / \mathcal{N}}$ be a densely defined projection such that $\mathcal{M} \subseteq \mathcal{N}^{\perp} \oplus \mathcal{N}$. By Lemma 3.5] $\mathcal{D}(E) \cap \mathcal{N}^{\perp}=$ $P_{\mathcal{N}^{\perp}}(\mathcal{M})$. In this case, the Moore-Penrose inverse $E^{\dagger}$ of $E$ is well defined (see [34]): $E^{\dagger}: \mathcal{M} \oplus \mathcal{M}^{\perp} \rightarrow$ $P_{\mathcal{N}^{\perp}}(\mathcal{M}) \subseteq P_{\mathcal{N}^{\perp}}(\mathcal{M}) \oplus \overline{\mathcal{N}}$

$$
E^{\dagger}= \begin{cases}0 & \text { if } x \in \mathcal{M}^{\perp} \\ \left(\left.E\right|_{P_{\mathcal{N}^{\perp}}(\mathcal{M})}\right)^{-1} x & \text { if } x \in \mathcal{M}\end{cases}
$$

The operators $E E^{\dagger}$ and $E^{\dagger} E$ are well defined and they are densely defined projections. In fact, $E E^{\dagger}=P_{\overline{\mathcal{M}}}$ on $\mathcal{D}\left(E^{\dagger}\right)=\mathcal{M} \oplus \mathcal{M}^{\perp}$ and $E^{\dagger} E=P_{\mathcal{N}^{\perp}}$ on $\mathcal{D}(E)=P_{\mathcal{N}^{\perp}}(\mathcal{M}) \oplus \mathcal{N}$. 
Proposition 3.7. Let $E=P_{\mathcal{M} / \mathcal{N}}$ be a densely defined projection such that $\mathcal{M} \subseteq \mathcal{N}^{\perp} \oplus \mathcal{N}$. Then

$$
E^{\dagger}=\left(P_{\mathcal{M} / \mathbb{N}}\right)^{\dagger}
$$

Proof. By Lemma 3.5, $\tilde{E}:=P_{\mathcal{M} / \mathbb{\mathcal { N }}}$ is a densely defined projection such that $E \subseteq \tilde{E}$. Since $\left.E\right|_{P_{\mathcal{N} \perp}(\mathcal{M})}=$ $\left.\tilde{E}\right|_{P_{\mathcal{N} \perp}(\mathcal{M})}$, it follows that $E^{\dagger}=\tilde{E}^{\dagger}$.

In view of Proposition 3.7, we focus our attention on the Moore-Penrose inverse of densely defined projections with closed nullspace.

Proposition 3.8. Let $E=P_{\mathcal{M} / \mathcal{N}}$ be a densely defined projection with closed nullspace. Then

$$
E^{\dagger}=P_{\mathcal{N} \perp} P_{\overline{\mathcal{M}}} \text { on } \mathcal{D}\left(E^{\dagger}\right)=\mathcal{M} \oplus \mathcal{M}^{\perp} .
$$

Proof. If $x \in \mathcal{M}^{\perp}$ then $E^{\dagger} x=0$. On the other hand, if $m \in \mathcal{M}$, let $y:=\left(\left.E\right|_{P_{\mathcal{N}^{\perp}}(\mathcal{M})}\right)^{-1} m$, then $y \in P_{\mathcal{N}^{\perp}}(\mathcal{M})$ and $E y=m$; also, $E P_{\mathcal{N}^{\perp}} m=E m=m$. Therefore

$$
E^{\dagger} m=\left(\left.E\right|_{P_{\mathcal{N}}(\mathcal{M})}\right)^{-1} m=P_{\mathcal{N}^{\perp}} m=P_{\mathcal{N}^{\perp}} P_{\overline{\mathcal{M}}} m .
$$

If $E=P_{\mathcal{M} / \mathcal{N}}$ is a densely defined closed projection, then $E^{\dagger}=P_{\mathcal{N}^{\perp}} P_{\mathcal{M}} \in \mathcal{P} \cdot \mathcal{P}$. Moreover, the map $E \mapsto E^{\dagger}$ from the set of densely defined closed projections onto $\mathcal{P} \cdot \mathcal{P}$ is a bijection, see [16].

To study the semiclosed case, consider the set

$$
\mathcal{P} \cdot L(\mathcal{H})^{+}:=\left\{T \in L(\mathcal{H}): T=P A \text { with } P \in \mathcal{P} \text { and } A \in L(\mathcal{H})^{+}\right\} .
$$

This set was studied in [7], where it was showed that any $T \in \mathcal{P} \cdot L(\mathcal{H})^{+}$can be factored as $T=P_{T} A$, where $P_{T}:=P_{\overline{\mathcal{R}(T)}}$ and $A \in L(\mathcal{H})^{+}$is such that $\mathcal{N}(T)=\mathcal{N}(A)$, though this factorization may not be unique. We say that $A \in L(\mathcal{H})^{+}$is optimal for $T$ if $T=P_{T} A$ and $\mathcal{N}(T)=\mathcal{N}(A)$. A description of the set of optimal operators for $T$ can be found in 7 , Remark 4.2 and Proposition 4.4].

Proposition 3.9. Let $E=P_{\mathcal{M} / \mathcal{N}} \in \mathcal{Q}_{S C}$. If $\mathcal{N}(E)$ is closed then there exists $\Gamma \in L(\mathcal{H})^{+}$such that $\mathcal{R}(\Gamma)=\mathcal{D}\left(E^{\dagger}\right)$ and $E^{\dagger} \Gamma \in \mathcal{P} \cdot L(\mathcal{H})^{+}$.

Proof. By Proposition 3.8, $E^{\dagger}=P_{\mathcal{N}^{\perp}} P_{\overline{\mathcal{M}}}$ on $\mathcal{D}\left(E^{\dagger}\right)$. If $A \in L(\mathcal{H})^{+}$is such that $\mathcal{R}(A)=\mathcal{M}$, take $\Gamma:=\left[\begin{array}{cc}A & 0 \\ 0 & I\end{array}\right] \quad \overline{\mathcal{M}}$. Then $\Gamma \in L(\mathcal{H})^{+}$and $\mathcal{R}(\Gamma)=\mathcal{R}(A) \oplus \mathcal{M}^{\perp}=\mathcal{D}\left(E^{\dagger}\right)$. Hence $E^{\dagger} \Gamma=P_{\mathcal{N}^{\perp}} P_{\overline{\mathcal{M}}} \Gamma=$ $P_{\mathcal{N} \perp} A \in \mathcal{P} \cdot L(\mathcal{H})^{+}$.

This generalizes the fact that if $E$ is a densely defined closed projection then $E^{\dagger} \in \mathcal{P} \cdot \mathcal{P}$, since in this case the operator $\Gamma$ can be chosen to be the identity. From Proposition 3.9 it follows that every $E \in \mathcal{Q}_{S C}$ with closed nullspace has an associated set in $\mathcal{P} \cdot L(\mathcal{H})^{+}$, namely, $\left\{T=P_{\mathcal{N}(E) \perp} A: A \in L(\mathcal{H})^{+}, \mathcal{R}(A)=\right.$ $\mathcal{R}(E)\}$.

On the other hand, every $T \in \mathcal{P} \cdot L(\mathcal{H})^{+}$has an associated set of semiclosed projections.

Proposition 3.10. Let $T \in \mathcal{P} \cdot L(\mathcal{H})^{+}$. If $A \in L(\mathcal{H})^{+}$is optimal for $T$ then

$$
\overline{\mathcal{R}(A) \dot{+} \mathcal{R}(T)^{\perp}}=\mathcal{H} \text {. }
$$

Proof. Consider $T \in \mathcal{P} \cdot L(\mathcal{H})^{+}$and write $T=P_{T} A$ with $A \in L(\mathcal{H})^{+}$optimal. Observe that $\mathcal{R}(A) \cap$ $\mathcal{R}(T)^{\perp}=\{0\}$. In fact, if $x \in \mathcal{R}(A) \cap \mathcal{R}(T)^{\perp}$ then $x=A y$ for some $y \in \mathcal{H}$ and $0=P_{T} x=P_{T} A y=T y$. Then $y \in \mathcal{N}(T)=\mathcal{N}(A)$ and $x=A y=0$. Also, $\mathcal{R}(A) \dot{+} \mathcal{R}(T)^{\perp}=P_{T}(\mathcal{R}(A)) \oplus \mathcal{R}(T)^{\perp}=\mathcal{R}(T) \oplus \mathcal{R}(T)^{\perp}$ is dense in $\mathcal{H}$.

Given $T \in \mathcal{P} \cdot L(\mathcal{H})^{+}$, define the set

$$
\Phi(T):=\left\{E=P_{\mathcal{R}(A) / / R(T)^{\perp}} \text { such that } A \text { is optimal for } T\right\} .
$$

By Proposition 3.10, every $E \in \Phi(T)$ is a densely defined semiclosed projection with closed nullspace. Moreover, $\mathcal{D}(E)=\mathcal{R}(T) \oplus \mathcal{R}(T)^{\perp}$ and $\overline{\mathcal{R}(E)}=\overline{\mathcal{R}\left(T^{*}\right)}$. Also, there exists a unique $E \in \Phi(T)$ if and only if $\overline{\mathcal{R}\left(T^{*}\right)} \cap \mathcal{N}\left(T^{*}\right)=\{0\}$, see [7, Proposition 4.1]. 


\section{B-symmetric projections}

A densely defined operator $T$ is symmetric if $T \subset T^{*}$ and it is selfadjoint if $T=T^{*}$, i.e., $T$ is symmetric and $\mathcal{D}(T)=\mathcal{D}\left(T^{*}\right)$.

Definition. Let $B \in L(\mathcal{H})^{s}$ and $E$ be a densely defined projection. We say that $E$ is $B$-symmetric if $B E$ is symmetric and it is $B$-selfadjoint if $B E$ is selfadjoint.

Since $B$ is bounded, $\mathcal{D}(B E)=\mathcal{D}(E)$ and $(B E)^{*}=E^{*} B$. Therefore $E$ is $B$-symmetric if and only if $B E x=E^{*} B x$ for every $x \in \mathcal{D}(E)$ and $E$ is $B$-selfadjoint if and only if $B E=E^{*} B$.

Proposition 4.1. Let $B \in L(\mathcal{H})^{s}$ and $E$ be a densely defined projection in $\mathcal{H}$. Then $E$ is $B$-symmetric if and only if $\mathcal{N}(E) \subseteq(B \mathcal{R}(E))^{\perp}$.

Proof. Suppose that $E$ is $B$-symmetric. Let $x \in \mathcal{N}(E)$ and $y \in \mathcal{D}(E)$. Then

$$
\langle x, B E y\rangle=\left\langle x, E^{*} B y\right\rangle=\langle E x, B y\rangle=0
$$

so, $\mathcal{N}(E) \subseteq(B \mathcal{R}(E))^{\perp}$. Conversely, suppose that $\mathcal{N}(E) \subseteq(B \mathcal{R}(E))^{\perp}=B^{-1}\left(\mathcal{N}\left(E^{*}\right)\right)$. Then $B \mathcal{N}(E) \subseteq$ $\mathcal{N}\left(E^{*}\right)$ and $B \mathcal{R}(E) \subseteq \overline{B \mathcal{R}(E)} \subseteq \mathcal{N}(E)^{\perp}=\mathcal{R}\left(E^{*}\right)$. Then $B \mathcal{D}(E)=B \mathcal{R}(E)+B \mathcal{N}(E) \subseteq \mathcal{R}\left(E^{*}\right) \dot{\dot{\phi}}$ $\mathcal{N}\left(E^{*}\right)=\mathcal{D}\left(E^{*}\right)$. Then $E^{*} B x=0$ for every $x \in \mathcal{N}(E)$ and $E^{*} B x=B x=B E x$ for every $x \in \mathcal{R}(E)$. Finally, if $x \in \mathcal{D}(E)$ then $B x, B E x, B(I-E) x \in \mathcal{D}\left(E^{*}\right)$ and $E^{*} B x=E^{*} B E x+E^{*} B(I-E) x=B E x$. Then $E$ is $B$-symmetric.

Proposition 4.2. Let $B \in L(\mathcal{H})^{s}$ and $\mathcal{S}$ a (not necessarily closed) subspace. There exists a $B$-symmetric projection onto $\mathcal{S}$ if and only if

$$
\mathcal{H}=\overline{\mathcal{S}+(B \mathcal{S})^{\perp}} .
$$

In this case, $\mathcal{S} \cap(B \mathcal{S})^{\perp}=\mathcal{S} \cap \mathcal{N}(B)$.

Proof. Suppose that $E$ is a $B$-symmetric projection onto $\mathcal{S}$. Then, by Proposition 4.1, $\mathcal{N}(E) \subseteq(B \mathcal{S})^{\perp}$ and $\mathcal{D}(E)=\mathcal{R}(E)+\mathcal{N}(E) \subseteq \mathcal{S}+(B \mathcal{S})^{\perp}$. Therefore $\mathcal{H}=\overline{\mathcal{D}(E)} \subseteq \overline{\mathcal{S}+(B \mathcal{S})^{\perp}}$.

Conversely, suppose that $\mathcal{H}=\overline{\mathcal{S}+(B \mathcal{S})^{\perp}}=\overline{\overline{\mathcal{S}}+(B \mathcal{S})^{\perp}}$. Let $\mathcal{L}^{\prime}:=\overline{\mathcal{S}} \cap(B \mathcal{S})^{\perp}$, then $\overline{\mathcal{S}}+(B \mathcal{S})^{\perp}=$ $\overline{\mathcal{S}} \dot{+}(B \mathcal{S})^{\perp} \cap \mathcal{L}^{\prime \perp}$, hence $\mathcal{S} \cap\left((B \mathcal{S})^{\perp} \cap \mathcal{L}^{\prime \perp}\right)=\{0\}$.

Define $E:=P_{\mathcal{S} /(B \mathcal{S})^{\perp} \cap \mathcal{L}^{\prime \perp}}$. Then $\overline{\mathcal{D}(E)}=\overline{\mathcal{S} \dot{+}\left((B \mathcal{S})^{\perp} \cap \mathcal{L}^{\perp}\right)}=\overline{\overline{\mathcal{S}} \dot{+}(B \mathcal{S})^{\perp} \cap \mathcal{L}^{\perp}}=\overline{\overline{\mathcal{S}}+(B \mathcal{S})^{\perp}}=$ $\mathcal{H}$. Therefore $E$ is a densely defined projection with (closed) nullspace contained in $(B \mathcal{S})^{\perp}$ and, by Proposition 4.1 $E$ is $B$-symmetric.

Finally, the inclusion $\mathcal{S} \cap \mathcal{N}(B) \subseteq \mathcal{S} \cap(B \mathcal{S})^{\perp}$ always holds. On the other hand, let $x \in \mathcal{S} \cap(B \mathcal{S})^{\perp}$ and $y \in \mathcal{D}(E)$ then

$$
\langle B x, y\rangle=\langle B E x, y\rangle=\langle x, B E y\rangle=0=\langle B x, E y\rangle .
$$

Then $B x \in \mathcal{D}(E)^{\perp}=\{0\}$ whence $x \in \mathcal{S} \cap \mathcal{N}(B)$.

When the projection $E$ is semiclosed, the $B$-symmetry can be given in terms of bounded operators.

Proposition 4.3. Let $B \in L(\mathcal{H})^{s}$ and $E \in \mathcal{Q}_{S C}$. Then $E$ is B-symmetric if and only if $P_{\Gamma}$ commutes with $\Gamma B \Gamma$, where $\Gamma \in L(\mathcal{H})^{+}$and $P_{\Gamma} \in \mathcal{P}$ are as in Corollary 3.4

Proof. Suppose that $E$ is $B$-symmetric, then $B \Gamma P_{\Gamma} x=B E \Gamma x=E^{*} B \Gamma x$ for every $x \in \mathcal{H}$. Then $\Gamma B \Gamma P_{\Gamma} x=\Gamma E^{*} B \Gamma x$ for every $x \in \mathcal{H}$. Now, since $E \Gamma=\Gamma P_{\Gamma}$, it follows that $\Gamma E^{*} \subset(E \Gamma)^{*}=P_{\Gamma}^{*} \Gamma$. Therefore $\Gamma B \Gamma P_{\Gamma} x=P_{\Gamma}^{*} \Gamma B \Gamma x$ for every $x \in \mathcal{H}$, i.e., $P_{\Gamma}$ is $\Gamma B \Gamma$-selfadjoint and since $P_{\Gamma}$ is selfadjoint, then $P_{\Gamma}$ commutes with $\Gamma B \Gamma$.

Conversely, if $P_{\Gamma}$ commutes with $\Gamma B \Gamma, \mathcal{N}\left(P_{\Gamma}\right) \subseteq(\Gamma B \Gamma)^{-1}\left(\mathcal{N}\left(P_{\Gamma}\right)\right)$. Therefore

$$
\mathcal{N}(E)=\Gamma\left(\mathcal{N}\left(P_{\Gamma}\right)\right) \subseteq B^{-1}\left(\mathcal{R}\left(\Gamma P_{\Gamma}\right)^{\perp}\right) \subseteq B^{-1}\left(\mathcal{R}(E)^{\perp}\right) .
$$

Then, by Proposition $4.1 E$ is $B$-symmetric. 


\section{Quasi and weak complementability}

The complementability of an operator $B \in L(\mathcal{H})$ with respect to two given closed subspaces $\mathcal{S}$ and $\mathcal{T}$ of $\mathcal{H}$ was studied for matrices by Ando [2] and Carlson and Haynsworth 13]. These ideas were extended to operators in Hilbert spaces in [17, 6].

Definition. Let $B \in L(\mathcal{H})^{s}$ and $\mathcal{S} \subseteq \mathcal{H}$ be a closed subspace. Then $B$ is $\mathcal{S}$-complementable if

$$
\mathcal{H}=\mathcal{S}+(B \mathcal{S})^{\perp} .
$$

In [17] it was shown that $B$ is $\mathcal{S}$-complementable if and only if there exists a $B$-selfadjoint projection onto $\mathcal{S}$; i.e., the set

$$
\mathcal{P}(B, \mathcal{S}):=\left\{Q \in \mathcal{Q}: \mathcal{R}(Q)=\mathcal{S}, B Q \in L(\mathcal{H})^{s}\right\}
$$

is not empty.

Proposition $5.1([17])$. Let $B \in L(\mathcal{H})^{s}$ and $\mathcal{S} \subseteq \mathcal{H}$ be a closed subspace. If the matrix decomposition of $B$ according to the orthogonal decomposition $\mathcal{H}=\mathcal{S} \oplus \mathcal{S}^{\perp}$ is given by

$$
B=\left[\begin{array}{cc}
a & b \\
b^{*} & c
\end{array}\right]
$$

then $B$ is $\mathcal{S}$-complementable if and only if $\mathcal{R}(b) \subseteq \mathcal{R}(a)$.

One way of generalizing the concept of complementability is to consider $B$-symmetric closed projections onto $\mathcal{S}$.

Definition. Let $B \in L(\mathcal{H})^{s}$ and $\mathcal{S} \subseteq \mathcal{H}$ be a closed subspace. We say that the pair $(B, \mathcal{S})$ is quasicomplementable if there exists a $B$-symmetric closed projection onto $\mathcal{S}$.

The set of quasi-complementable pairs was studied in [15] for a positive weight $B$. Many results stated in [15] hold also in the selfadjoint case.

Proposition 5.2. Let $B \in L(\mathcal{H})^{s}$ and $\mathcal{S} \subseteq \mathcal{H}$ be a closed subspace. The pair $(B, \mathcal{S})$ is quasicomplementable if and only if $\overline{B \mathcal{S}} \cap \mathcal{S}^{\perp}=\{0\}$.

Proof. $\quad$ It follows from the definition of quasi-complementability and Proposition 4.2

Proposition 5.3. Let $B \in L(\mathcal{H})^{s}, \mathcal{S} \subseteq \mathcal{H}$ be a closed subspace and $E$ be a densely defined closed projection onto $\mathcal{S}$. Then $E$ is $B$-symmetric if and only if

$$
a x \subset b,
$$

where $a, b$ are as in (5.1) and $x$ is as in (3.1).

Proof. The result follows by using arguments similar to those found in [15, Proposition 2.2].

Corollary 5.4. Let $\mathcal{S} \subseteq \mathcal{H}$ be a closed subspace and $B \in L(\mathcal{H})^{s}$ with representation as in (5.1). Then $(B, \mathcal{S})$ is quasi-complementable if and only if the equation

$$
b^{*}=x a
$$

admits a densely defined closed solution $x_{0}: \mathcal{D}\left(x_{0}\right) \subseteq \mathcal{S} \rightarrow \mathcal{S}^{\perp}$ with densely defined adjoint.

Proof. If $(B, \mathcal{S})$ is quasi-complementable then there exists $E$ a $B$-symmetric closed projection onto $\mathcal{S}$. Suppose that the matrix decomposition of $E$ is as in (3.1). Then, by Proposition 5.3, $a x \subset b$. Then $b^{*} \subset(a x)^{*}=x^{*} a$. Therefore, $b^{*}=x^{*} a$ and $x^{*}$ is a densely defined closed solution of (5.2) with densely defined adjoint. 
Conversely, let $x_{0}: \mathcal{D}\left(x_{0}\right) \subseteq \mathcal{S} \rightarrow \mathcal{S}^{\perp}$ be a densely defined closed solution of (5.2) with densely defined adjoint. Then $b=\left(x_{0} a\right)^{*} \supset a x_{0}^{*}$. Set $E:=\left[\begin{array}{cc}I & x_{0}^{*} \\ 0 & 0\end{array}\right]$, then $E$ is a densely defined closed projection with range $\mathcal{S}$ and, by Proposition [5.3, $E$ is $B$-symmetric. Hence, the pair $(B, \mathcal{S})$ is quasi-complementable.

A different way of extending the concept of complementability was given in [6], where Antezana et al. defined the notion of weak complementability to study the Schur complement in this context. We use these ideas when $\mathcal{S}=\mathcal{T}$ and $B \in L(\mathcal{H})^{s}$.

Definition. Let $\mathcal{S} \subseteq \mathcal{H}$ be a closed subspace, and $B \in L(\mathcal{H})^{s}$ with representation as in (5.1). Then $B$ is $\mathcal{S}$-weakly complementable if

$$
\mathcal{R}(b) \subseteq \mathcal{R}\left(|a|^{1 / 2}\right) .
$$

The notion of weak complementability is distinct to the notion of complementability only in the infinite dimensional setting. Every positive operator $B$ is $\mathcal{S}$-weakly complementable, and if $B$ is $\mathcal{S}$ weakly complementable for every closed subspace $\mathcal{S} \subseteq \mathcal{H}$ then $B$ is semidefinite, see 14, Proposition $3.1]$.

The next proposition gives an operator characterization of the $\mathcal{S}$-weak complementability as the solution of a Riccati type equation [5].

Proposition 5.5. Let $B \in L(\mathcal{H})^{s}$ and $\mathcal{S}$ be a closed subspace of $\mathcal{H}$. Then $B$ is $\mathcal{S}$-weakly complementable if and only if there exists a positive solution of the equation

$$
B P_{\mathcal{S}} B=X P_{\mathcal{S}} X^{*} .
$$

Proof. Suppose that the matrix decomposition of $B$ induced by $\mathcal{S}$ is as in (5.1) and $a=u|a|$ is the polar decomposition of $a$. Let $f \in L\left(\mathcal{S}^{\perp}, \mathcal{S}\right)$ be the reduced solution of $b=|a|^{1 / 2} x$.

Let $A:=\left[\begin{array}{cc}|a| & u|a|^{1 / 2} f \\ f^{*}|a|^{1 / 2} u & f^{*} f\end{array}\right]$. Then, by Lemma [2.3, $A \geq 0$, because $u f$ is the reduced solution of $|a|^{1 / 2} x=u|a|^{1 / 2} f$ and $f^{*} f=f^{*} u u f$. It easily follows that $B P_{\mathcal{S}} B=A P_{\mathcal{S}} A$.

Conversely, suppose that $B P_{\mathcal{S}} B=A P_{\mathcal{S}} A$ with $A \geq 0$ and $A=\left[\begin{array}{ll}a_{11} & a_{12} \\ a_{12}^{*} & a_{22}\end{array}\right]$. Then,

$$
\begin{array}{r}
a^{2}=a_{11}^{2} \\
a b=a_{11} a_{12} \\
b^{*} b=a_{12}^{*} a_{12} .
\end{array}
$$

From (5.3), $a_{11}=|a|$. Thus, by (5.4), $u a_{11} b=a b=a_{11} a_{12}$. Then $a_{11} b=u a_{11} a_{12}=a_{11} u a_{12}$. So that

$$
a_{11}^{1 / 2}\left(b-u a_{12}\right)=0=\left(b-u a_{12}\right)^{*} a_{11}^{1 / 2} .
$$

Then, from (5.5),$a_{12}^{*} a_{12}=b^{*} b=\left(b-u a_{12}+u a_{12}\right)^{*}\left(b-u a_{12}+u a_{12}\right)=\left(b-u a_{12}\right)^{*}\left(b-u a_{12}\right)+a_{12}^{*} a_{12}$. The last equality follows from Lemma 2.3, in fact, if $a_{12}=a_{11}^{1 / 2} g$, then $u a_{12}=u a_{11}^{1 / 2} g=a_{11}^{1 / 2} u g$, because $u$ and $a_{11}^{1 / 2}$ commute. Then $\left(b-u a_{12}\right)^{*} u a_{12}=\left(b-u a_{12}\right)^{*} a_{11}^{1 / 2} u g=0$. Therefore,

$$
b^{*} b=\left(b-u a_{12}\right)^{*}\left(b-u a_{12}\right)+b^{*} b .
$$

So that $\left|b-u a_{12}\right|=0$ or $b=u a_{12}=a_{11}^{1 / 2} u g$ and $\mathcal{R}(b) \subseteq \mathcal{R}\left(a_{11}^{1 / 2}\right)=\mathcal{R}\left(|a|^{1 / 2}\right)$.

Let $\mathcal{S}$ be a closed subspace of $\mathcal{H}$ and let $E$ be a densely defined projection with $\mathcal{N}(E)=\mathcal{S}^{\perp}$. Then, by Proposition 3.6. the matrix representation of $E$ according to the orthogonal decomposition $\mathcal{H}=\mathcal{S} \oplus \mathcal{S}^{\perp}$ is

$$
E=\left[\begin{array}{ll}
I & 0 \\
y & 0
\end{array}\right] \text { where } \mathcal{D}(E)=\mathcal{D}(y) \oplus \mathcal{S}^{\perp},
$$

with $\mathcal{D}(y)=P_{\mathcal{S}}(\mathcal{R}(E))$ dense in $\mathcal{S}$ and $y=P_{\mathcal{S}^{\perp}}\left(\left.P_{\mathcal{S}} P_{\overline{\mathcal{R}(E)}}\right|_{\mathcal{R}(E)}\right)^{-1}$.

To characterize the $\mathcal{S}$-weak complementability of $B$ in terms of projections we need the following lemma. 
Lemma 5.6. Let $B \in L(\mathcal{H})^{s}, \mathcal{S}$ be a closed subspace of $\mathcal{H}$ and $E$ be a densely defined projection with $\mathcal{N}(E)=\mathcal{S}^{\perp}$. Suppose that the matrix decomposition of $B$ and $E$ are as in (5.1) and (5.6), respectively. Then $E B \in L(\mathcal{H})^{s}$ if and only if ya $=b^{*}$ and $y b \in L\left(\mathcal{S}^{\perp}\right)^{s}$. Moreover, $B \mathcal{S} \subseteq \mathcal{R}(E)$ if and only if ya $=b^{*}$.

Proof. Suppose that $E B \in L(\mathcal{H})^{s}$. Then $E B=\left[\begin{array}{cc}a & b \\ y a & y b\end{array}\right]=(E B)^{*}=\left[\begin{array}{cc}a & (y a)^{*} \\ b^{*} & (y b)^{*}\end{array}\right]$. Therefore $y a=b^{*}$ and $y b \in L\left(\mathcal{S}^{\perp}\right)^{s}$. The converse follows in a similar way.

Moreover, if $B \mathcal{S} \subseteq \mathcal{R}(E)$ then

$$
0=(I-E) B s=\left[\begin{array}{cc}
0 & 0 \\
-y & I
\end{array}\right]\left[\begin{array}{cc}
a & b \\
b^{*} & c
\end{array}\right]\left[\begin{array}{l}
s \\
0
\end{array}\right]=-y a s+b^{*} s \text { for every } s \in \mathcal{S} .
$$

Then $b^{*}=y a$.

Conversely, if $b^{*}=y a$ then $B \mathcal{S} \subseteq \mathcal{R}(a)+\mathcal{R}\left(b^{*}\right) \subseteq \mathcal{D}(y) \oplus \mathcal{S}^{\perp}=\mathcal{D}(E)=\mathcal{D}(I-E)$ and $(I-E) B s=$ - yas $+b^{*} s=0$ for every $s \in \mathcal{S}$. Hence, $B \mathcal{S} \subseteq \mathcal{R}(E)$.

Corollary 5.7. Let $B \in L(\mathcal{H})^{s}, \mathcal{S}$ be a closed subspace of $\mathcal{H}$ and $E$ be a densely defined projection with $\mathcal{N}(E)=\mathcal{S}^{\perp}$. If $E B \in L(\mathcal{H})^{s}$ then $B \mathcal{S} \cap \mathcal{S}^{\perp}=\{0\}$.

Corollary 5.8. Let $B \in L(\mathcal{H})^{s}, \mathcal{S}$ be a closed subspace of $\mathcal{H}$ and $E \in \mathcal{Q}_{S C}$ with $\mathcal{N}(E)=\mathcal{S}^{\perp}$. Suppose that the matrix decomposition of $B$ and $E$ are as in (5.1) and (5.6), respectively. Then $E B \in L(\mathcal{H})^{s}$ and $\mathcal{R}\left(|a|^{1 / 2}\right) \subseteq \mathcal{D}(E)$ if and only if $y a=b^{*}$ and $\mathcal{R}\left(|a|^{1 / 2}\right) \subseteq \mathcal{D}(y)$.

Proof. Suppose that $E B \in L(\mathcal{H})^{s}$ and $\mathcal{R}\left(|a|^{1 / 2}\right) \subseteq \mathcal{D}(E)$. Then, by Lemma 5.6 $y a=b^{*}$. Also, since $\mathcal{D}(E)=\mathcal{D}(y) \oplus \mathcal{S}^{\perp}$, it follows that $\mathcal{R}\left(|a|^{1 / 2}\right) \subseteq \mathcal{D}(y)$.

Conversely, suppose that $y a=b^{*}$ and $\mathcal{R}\left(|a|^{1 / 2}\right) \subseteq \mathcal{D}(y)$. Then $\mathcal{R}\left(|a|^{1 / 2}\right) \subseteq \mathcal{D}(y) \oplus \mathcal{S}^{\perp}=\mathcal{D}(E)$. By Corollary 2.7. $E|a|^{1 / 2} \in L(\mathcal{S}, \mathcal{H})$, so that $y|a|^{1 / 2} \in L\left(\mathcal{S}, \mathcal{S}^{\perp}\right)$. Then, if $a=u|a|$ is the polar decomposition of $a$,

$$
y b=y(y a)^{*}=y\left(y|a|^{1 / 2} u|a|^{1 / 2}\right)^{*}=y|a|^{1 / 2} u\left(y|a|^{1 / 2}\right)^{*} .
$$

Hence $y b \in L\left(\mathcal{S}^{\perp}\right)^{s}$ and, by Lemma 5.6, $E B \in L(\mathcal{H})^{s}$.

The next theorem characterizes the $\mathcal{S}$-weak complementability of a selfadjoint operator in terms of semiclosed projections. See also [14, Theorem 3.14].

Theorem 5.9. Let $B \in L(\mathcal{H})^{s}$ and $\mathcal{S}$ be a closed subspace of $\mathcal{H}$. Suppose that the matrix decomposition of $B$ is as in (5.1). Then $B$ is $\mathcal{S}$-weakly complementable if and only if there exists $E \in \mathcal{Q}_{S C}$ with $\mathcal{N}(E)=\mathcal{S}^{\perp}$ such that $E B \in L(\mathcal{H})^{s}$ and $\mathcal{R}\left(|a|^{1 / 2}\right) \subseteq \mathcal{D}(E)$.

Proof. Suppose that $B$ is $\mathcal{S}$-weakly complementable. If the matrix decomposition of $B$ induced by $\mathcal{S}$ is as in (5.1), let $f$ be the reduced solution of $b=|a|^{1 / 2} x$ and $a=u|a|$ be the polar decomposition of $a$. Write $\left(|a|^{1 / 2}\right)^{\dagger}$ for the Moore-Penrose inverse of $|a|^{1 / 2}$ and set

$$
E:=\left[\begin{array}{cc}
I & 0 \\
f^{*} u\left(|a|^{1 / 2}\right)^{\dagger} & 0
\end{array}\right]
$$

Then $\mathcal{D}(E)=\mathcal{D}\left(\left(|a|^{1 / 2}\right)^{\dagger}\right) \oplus \mathcal{S}^{\perp}$ is a semiclosed subspace of $\mathcal{H}$ (because it is the sum of two semiclosed subspaces), $E$ is a densely defined projection with $\mathcal{N}(E)=\mathcal{S}^{\perp}$ and $\mathcal{R}\left(|a|^{1 / 2}\right) \subseteq \mathcal{D}(E)$. On the other hand, since $\mathcal{R}(B) \subseteq \mathcal{R}\left(|a|^{1 / 2}\right) \oplus \mathcal{S}^{\perp}$, the product $(I-E) B$ is well defined. Moreover,

$$
\begin{aligned}
(I-E) B & =\left[\begin{array}{cc}
0 & 0 \\
-f^{*} u\left(|a|^{1 / 2}\right)^{\dagger} & I
\end{array}\right]\left[\begin{array}{cc}
|a|^{1 / 2} u|a|^{1 / 2} & |a|^{1 / 2} f \\
f^{*}|a|^{1 / 2} & c
\end{array}\right] \\
& =\left[\begin{array}{cc}
0 & 0 \\
0 & c-f^{*} u f
\end{array}\right] \in L(\mathcal{H})^{s} .
\end{aligned}
$$

So that $E B \in L(\mathcal{H})^{s}$.

$$
\text { Finally, if } \Gamma:=\left[\begin{array}{cc}
|a|^{1 / 2}+P_{\mathcal{N}(a)} & 0 \\
0 & I
\end{array}\right] \text {, then } \Gamma \in L(\mathcal{H})^{+}, \mathcal{R}(\Gamma)=\mathcal{D}(E) \text { and }
$$




$$
E \Gamma=\left[\begin{array}{cc}
|a|^{1 / 2}+P_{\mathcal{N}(a)} & 0 \\
f^{*} u & 0
\end{array}\right] \in L(\mathcal{H}) .
$$

By Corollary 2.6, $E$ is semiclosed.

Conversely, suppose that there exists $E \in \mathcal{Q}_{S C}$ with $\mathcal{N}(E)=\mathcal{S}^{\perp}$ such that $E B \in L(\mathcal{H})^{s}$ and $\mathcal{R}\left(|a|^{1 / 2}\right) \subseteq \mathcal{D}(E)$. Suppose that the matrix decomposition of $E$ is that as in (5.6). By Corollary 2.7 $E|a|^{1 / 2} \in L(\mathcal{S}, \mathcal{H})$, and then $y|a|^{1 / 2} \in L\left(\mathcal{S}, \mathcal{S}^{\perp}\right)$. By Lemma [5.6 $y a=b^{*}$ and $y b \in L\left(\mathcal{S}^{\perp}\right)^{s}$. Since $y a=b^{*}$, we also have that $y|a|^{1 / 2} u|a|^{1 / 2}=b^{*}$. Then $b=|a|^{1 / 2} u\left(y|a|^{1 / 2}\right)^{*}, \mathcal{R}(b) \subseteq \mathcal{R}\left(|a|^{1 / 2}\right)$ and $B$ is $\mathcal{S}$-weakly complementable.

Suppose that $B$ is $\mathcal{S}$-weakly complementable. If the matrix decomposition of $B$ induced by $\mathcal{S}$ is as in (5.1), let $f$ be the reduced solution of $b=|a|^{1 / 2} x$ and $a=u|a|$ be the polar decomposition of $a$. Set

$$
E_{0}:=\left[\begin{array}{cc}
I & 0 \\
y_{0} & 0
\end{array}\right]
$$

with $y_{0}:=f^{*} u\left(|a|^{1 / 2}\right)^{\dagger}$. Then, by the proof of Theorem 5.9 $E_{0} \in \mathcal{Q}_{S C}$ with $\mathcal{N}\left(E_{0}\right)=\mathcal{S}^{\perp}, E_{0} B \in L(\mathcal{H})^{s}$ and $\mathcal{D}\left(E_{0}\right)=\mathcal{D}\left(\left(|a|^{1 / 2}\right)^{\dagger}\right) \oplus \mathcal{S}^{\perp}$. Define

$$
\mathcal{P}^{*}(B, \mathcal{S}):=\left\{E \in \mathcal{Q}_{S C}: \mathcal{N}(E)=\mathcal{S}^{\perp}, E B \in L(\mathcal{H})^{s} \text { and } \mathcal{R}\left(|a|^{1 / 2}\right) \subseteq \mathcal{D}(E)\right\} .
$$

Let $E \in \mathcal{P}^{*}(B, \mathcal{S})$ with matrix decomposition as in (5.6). Then, it can be proved that

$$
\mathcal{R}\left(y_{0}\right) \subseteq \mathcal{R}(y) .
$$

Furthermore, $y|a|^{1 / 2}=y_{0}|a|^{1 / 2}$.

In what follows, we characterize the subset of projections with fixed domain $\mathcal{D}\left(E_{0}\right)$. More precisely, consider

$$
\mathcal{P}_{0}^{*}(B, \mathcal{S}):=\left\{E \in \mathcal{P}^{*}(B, \mathcal{S}): \mathcal{D}(E)=\mathcal{D}\left(E_{0}\right)\right\} .
$$

From Lemma 5.6 and Corollary 5.8, $\mathcal{P}_{0}^{*}(B, \mathcal{S})=\left\{E \in \mathcal{Q}_{S C}: \mathcal{D}(E)=\mathcal{D}\left(E_{0}\right), \mathcal{N}(E)=\mathcal{S}^{\perp}\right.$ and $B \mathcal{S} \subseteq$ $\mathcal{R}(E)\}$.

Clearly, $\mathcal{P}_{0}^{*}(B, \mathcal{S}) \subseteq \mathcal{P}^{*}(B, \mathcal{S})$ and $\mathcal{P}_{0}^{*}(B, \mathcal{S})$ is not empty because $E_{0} \in \mathcal{P}_{0}^{*}(B, \mathcal{S})$.

Theorem 5.10. Let $B \in L(\mathcal{H})^{s}$ and $\mathcal{S}$ be a closed subspace of $\mathcal{H}$ such that $B$ is $\mathcal{S}$-weakly complementable. Then

$$
\mathcal{P}_{0}^{*}(B, \mathcal{S})=E_{0}+\left\{W \in S C(\mathcal{H}): \mathcal{D}\left(E_{0}\right) \subseteq \mathcal{D}(W), \mathcal{R}(W) \subseteq \mathcal{S}^{\perp} \text { and } B \mathcal{S}+\mathcal{S}^{\perp} \subseteq \mathcal{N}(W)\right\}
$$

Proof. Let $E=E_{0}+W$, with $W \in S C(\mathcal{H})$ such that $\mathcal{D}\left(E_{0}\right) \subseteq \mathcal{D}(W), \mathcal{R}(W) \subseteq \mathcal{S}^{\perp}$ and $B \mathcal{S}+\mathcal{S}^{\perp} \subseteq$ $\mathcal{N}(W)$. Then $E_{0}+W \in S C(\mathcal{H})$ and $\mathcal{D}\left(E_{0}+W\right)=\mathcal{D}\left(E_{0}\right) \cap \mathcal{D}(W)=\mathcal{D}\left(E_{0}\right)$. Observe that, $\mathcal{R}\left(E_{0}+W\right) \subseteq$ $\mathcal{R}\left(E_{0}\right)+\mathcal{S}^{\perp}=\mathcal{D}\left(E_{0}\right)=\mathcal{D}\left(E_{0}+W\right)$. Also, since $\mathcal{R}(W) \subseteq \mathcal{N}(W) \cap \mathcal{N}\left(E_{0}\right), W^{2}=0, E_{0} W=0$ and, from $\mathcal{R}\left(I-E_{0}\right) \subseteq \mathcal{N}(W), W E_{0}=W$. Hence $E_{0}+W \in \mathcal{Q}_{S C}$. Furthermore, $\mathcal{N}\left(E_{0}+W\right)=\mathcal{S}^{\perp}$. In fact, it is clear that $\mathcal{S}^{\perp} \subseteq \mathcal{N}\left(E_{0}+W\right)$ and if $h \in \mathcal{N}\left(E_{0}+W\right) \subseteq \mathcal{D}\left(E_{0}+W\right)=\mathcal{D}\left(E_{0}\right)$ then $E_{0} h=-W h$, so that $E_{0} h \in \mathcal{R}\left(E_{0}\right) \cap \mathcal{R}(W) \subseteq \mathcal{R}\left(E_{0}\right) \cap \mathcal{S}^{\perp}=\{0\}$. Therefore $h \in \mathcal{N}\left(E_{0}\right)=\mathcal{S}^{\perp}$ and $\mathcal{N}\left(E_{0}+W\right) \subseteq \mathcal{S}^{\perp}$. Also, $\mathcal{R}(B) \subseteq \mathcal{D}\left(E_{0}\right)=\mathcal{D}\left(E_{0}+W\right)$ and $B \mathcal{S} \subseteq \mathcal{R}\left(E_{0}+W\right)$. In fact, if $h \in B \mathcal{S}$, since $E_{0} B \in L(\mathcal{H})^{s}$, by Lemma 5.6. $h \in \mathcal{R}\left(E_{0}\right) \subseteq \mathcal{D}\left(E_{0}\right)=\mathcal{D}\left(W+E_{0}\right)$. Hence, $\left(E_{0}+W\right) h=E_{0} h+W h=h$, because $h \in \mathcal{N}(W)$. Then $E_{0}+W \in \mathcal{P}_{0}^{*}(B, \mathcal{S})$.

Conversely, let $E \in \mathcal{P}_{0}^{*}(B, \mathcal{S})$ and define $W:=E-E_{0}$. Then $W \in S C(\mathcal{H})$ and $\mathcal{D}(W)=\mathcal{D}(E) \cap$ $\mathcal{D}\left(E_{0}\right)=\mathcal{D}\left(E_{0}\right)$. Also, $\mathcal{R}(W)=\mathcal{R}\left(\left(I-E_{0}\right)+(E-I)\right) \subseteq \mathcal{S}^{\perp}$. It is clear that $\mathcal{S}^{\perp} \subseteq \mathcal{N}(W)$ and, if $h \in B \mathcal{S}$, by Lemma 5.6. $h \in \mathcal{R}(E) \cap \mathcal{R}\left(E_{0}\right) \subseteq \mathcal{D}\left(E_{0}\right)$. Then $W h=E h-E_{0} h=h-h=0$. Therefore $B \mathcal{S}+\mathcal{S}^{\perp} \subseteq \mathcal{N}(W)$ and $E=E_{0}+W$. 


\subsection{Comparison between the notions of quasi and weak complementability}

The next examples show that quasi-complementability does not imply weak complementability and viceversa.

Example 1. Let $\mathcal{S} \subseteq \mathcal{H}$ be a closed subspace such that $\operatorname{dim}(\mathcal{S})=\operatorname{dim}\left(\mathcal{S}^{\perp}\right)=\infty$. Take $a \in L(\mathcal{S})^{+}$ such that $\mathcal{R}(a)$ is not closed and $\overline{\mathcal{R}(a)}=\mathcal{S}$, and take $b: \mathcal{S}^{\perp} \rightarrow \mathcal{S}$ such that $b$ is invertible. Consider $B=\left[\begin{array}{cc}a & b \\ b^{*} & c\end{array}\right]$ for any $c \in L\left(\mathcal{S}^{\perp}\right)^{s}$. Then $B$ is not $\mathcal{S}$-weakly complementable because $\mathcal{R}(b)=\mathcal{S} \nsubseteq \mathcal{R}\left(a^{1 / 2}\right)$.

Now, we want to show that $\overline{B \mathcal{S}} \cap \mathcal{S}^{\perp}=\{0\}$. Let $h \in \overline{B \mathcal{S}} \cap \mathcal{S}^{\perp}$. Then there exists $\left\{s_{n}\right\}_{n \geq 1} \subseteq \mathcal{S}$ such that

$$
h=\lim _{n \rightarrow \infty} B s_{n}=\lim _{n \rightarrow \infty}\left(a s_{n}+b^{*} s_{n}\right)=\lim _{n \rightarrow \infty} P_{\mathcal{S}^{\perp}}\left(a s_{n}+b^{*} s_{n}\right)=\lim _{n \rightarrow \infty} b^{*} s_{n}
$$

Then $\left(b^{*}\right)^{-1} h=\lim _{n \rightarrow \infty} s_{n}$ and $0=\lim _{n \rightarrow \infty} a s_{n}$. Therefore $a\left(b^{*}\right)^{-1} h=0$. Since $a$ is injective it follows that $\left(b^{*}\right)^{-1} h=0$ then $h=0$. Hence, by Proposition [5.2, the pair $(B, \mathcal{S})$ is quasi-complementable.

Example 2. See [15, Example 2.14]. Let $B \in L(\mathcal{H})^{+}$be such that $\mathcal{R}(B)$ is not closed. Let $x \in \overline{\mathcal{R}(B)} \backslash$ $\mathcal{R}(B)$ and define the closed subspace $\mathcal{S}$ such that $\mathcal{S}^{\perp}=\operatorname{span}\{x\}$. Clearly, $B$ is $\mathcal{S}$-weakly complementable. On the other hand, $(B \mathcal{S})^{\perp}=B^{-1}\left(\mathcal{S}^{\perp}\right)=B^{-1}(\operatorname{span}\{x\})=B^{-1}(\operatorname{span}\{x\} \cap \mathcal{R}(B))=B^{-1}(\{0\})=\mathcal{N}(B)$. Then $\overline{B S}=\overline{\mathcal{R}(B)}$ and $\mathcal{S}^{\perp} \cap \overline{B \mathcal{S}} \neq\{0\}$. Therefore, by Proposition [5.2, the pair $(B, \mathcal{S})$ is not quasi complementable.

Let $B \in L(\mathcal{H})^{s}$ and let $\mathcal{S}$ be a closed subspace of $\mathcal{H}$ and suppose that $\mathcal{S}=\mathcal{S}_{+} \oplus_{B} \quad \mathcal{S}_{-}$is any decomposition as in (2.2). In [14, Proposition 3.2] it was shown that $B$ is $\mathcal{S}$-weakly complementable if and only if there exist $B_{1}, B_{2}, B_{3} \in L(\mathcal{H})^{s}$, such that

$$
B=B_{1}+B_{2}-B_{3},
$$

where $B_{2}, B_{3} \geq 0, \mathcal{S} \subseteq \mathcal{N}\left(B_{1}\right), \mathcal{S}_{-} \subseteq \mathcal{N}\left(B_{2}\right), \mathcal{S}_{+} \subseteq \mathcal{N}\left(B_{3}\right)$

In the following proposition we characterize the $B$-symmetric closed projections onto $\mathcal{S}$ for $B \in L(\mathcal{H})^{s}$ when $B$ is $\mathcal{S}$-weakly complementable. Some of these results where stated in [15] for a positive weight $B$. To extend these results to the selfadjoint case the notion of semiclosed projections turns out to be useful.

Proposition 5.11. Let $B \in L(\mathcal{H})^{s}, E$ be a densely defined closed projection of $\mathcal{H}$ onto $\mathcal{S}$ and suppose that $B$ is $\mathcal{S}$-weakly complementable. If $E$ is $B$-symmetric, then $B E$ admits a bounded selfadjoint extension to $\mathcal{H}$. Moreover, if $\mathcal{S}=\mathcal{S}_{1} \oplus_{B} \mathcal{S}_{2}$ and $B=B_{1}+B_{2}-B_{3}$, are any decompositions as in (2.2) and (5.8), respectively, then

$$
\overline{B E}=(B E)^{*}=B_{2}^{1 / 2} P_{\mathcal{M}_{2}} B_{2}^{1 / 2}-B_{3}^{1 / 2} P_{\mathcal{M}_{3}} B_{3}^{1 / 2} \in L(\mathcal{H})^{s},
$$

where $\mathcal{M}_{2}=\overline{B_{2}^{1 / 2}\left(\mathcal{S}_{+}\right)}$and $\mathcal{M}_{3}=\overline{B_{3}^{1 / 2}\left(\mathcal{S}_{-}\right)}$.

To prove this proposition we need the following lemma.

Lemma 5.12. Let $B \in L(\mathcal{H})^{s}$ and $E$ be a densely defined closed projection of $\mathcal{H}$ onto $\mathcal{S}$. Suppose that $B$ is $\mathcal{S}$-weakly complementable, $\mathcal{S}=\mathcal{S}_{+} \oplus_{B} \mathcal{S}_{-}$is any decomposition as in (2.2) and $B=B_{1}+B_{2}-B_{3}$ is any decomposition as in (5.8). If $E$ is $B$-symmetric, then $E$ admits a factorization $E=E_{+}+E_{-}$, where $E_{+}$and $E_{-}$are semiclosed projections, with $\mathcal{D}(E)=\mathcal{D}\left(E_{+}\right)=\mathcal{D}\left(E_{-}\right), \mathcal{R}\left(E_{+}\right)=\mathcal{S}_{+}, \mathcal{R}\left(E_{-}\right)=\mathcal{S}_{-}$, $E_{-} E_{+}=E_{+} E-=0, E_{+}$is $B_{2}$-symmetric and $E_{-}$is $B_{3}$-symmetric.

Proof. Since $E$ is $B$-symmetric, by Proposition 4.1, $E=P_{\mathcal{S} / / \mathcal{T}}$, with $\mathcal{T}$ a closed subspace such that $\mathcal{T} \subseteq B^{-1}\left(\mathcal{S}^{\perp}\right)$ and $\mathcal{D}(E)=\mathcal{S} \dot{+} \mathcal{T}=\mathcal{S}_{+} \dot{+} \mathcal{S}_{-} \dot{+} \mathcal{T}$. Let $E_{+}:=P_{\mathcal{S}_{+} / / \mathcal{T}+S_{-}}$and $E_{-}:=P_{\mathcal{S}_{-} / / \mathcal{T}+S_{+}}$. Then $E_{ \pm}=P_{\mathcal{S}_{ \pm}} E$ and $E_{ \pm}$are $B$-symmetric. Let us prove these assertions for $E_{+}$; the other case is similar. First observe that $\mathcal{D}\left(P_{\mathcal{S}_{+}} E\right)=\mathcal{D}(E)$ and $\mathcal{R}\left(P_{\mathcal{S}_{+}} E\right) \subseteq \mathcal{S}_{+} \subseteq \mathcal{D}(E)$. Then $\left(P_{\mathcal{S}_{+}} E\right)^{2}=P_{\mathcal{S}_{+}} E P_{\mathcal{S}_{+}} E=P_{\mathcal{S}_{+}} E$ so that $P_{\mathcal{S}_{+}} E$ is a densely defined projection. On the other hand, $\mathcal{R}\left(P_{\mathcal{S}_{+}} E\right)=P_{\mathcal{S}_{+}} \mathcal{R}(E)=\mathcal{S}_{+}$and $\mathcal{N}\left(P_{\mathcal{S}_{+}} E\right)=\mathcal{N}(E)+\mathcal{S} \cap \mathcal{S}_{+}^{\perp}=\mathcal{T}+\mathcal{S}_{-}$(see Lemma 2.2). Therefore $E_{+}=P_{\mathcal{S}_{+}} E$ and, since $\mathcal{N}\left(E_{+}\right)$and $\mathcal{R}\left(E_{+}\right)$are semiclosed subspaces, $E_{+}$is a semiclosed projection. Also, $\mathcal{D}(E)=\mathcal{D}\left(E_{+}\right)=\mathcal{D}\left(E_{-}\right)$and $E=P_{\mathcal{S}} E=P_{\mathcal{S}_{+}} E+P_{\mathcal{S}_{-}} E=E_{+}+E_{-}$with $E_{+} E_{-}=E_{-} E_{+}=0$. 
Now, let us see that $E_{+}$is $B$-symmetric. Note that $P_{\mathcal{S}} E=E$, so that $E^{*} P_{\mathcal{S}}=E^{*}$. Let $x \in \mathcal{D}\left(E_{+}\right)=$ $\mathcal{D}(E)$, since $E_{+} x \in \mathcal{D}(E)$,

$$
\begin{aligned}
B E_{+} x & =B E E_{+} x=E^{*} B E_{+} x=E^{*} B P_{\mathcal{S}_{+}} E x=E^{*} P_{\mathcal{S}} B P_{\mathcal{S}_{+}} E x=E^{*} P_{\mathcal{S}} B P_{\mathcal{S}} P_{\mathcal{S}_{+}} E x \\
& =E^{*} G_{B, \mathcal{S}} P_{\mathcal{S}_{+}} E x=E^{*} P_{\mathcal{S}_{+}} G_{B, \mathcal{S}} E x=E^{*} P_{\mathcal{S}_{+}} P_{\mathcal{S}} B E x=E^{*} P_{\mathcal{S}_{+}} B E x \\
& =E^{*} P_{\mathcal{S}_{+}} E^{*} B x=\left(P_{\mathcal{S}_{+}} E\right)^{*} E^{*} B x=E_{+}^{*} E^{*} B x=E_{+}^{*} B x,
\end{aligned}
$$

where we used that $G_{B, \mathcal{S}} P_{\mathcal{S}_{+}}=P_{\mathcal{S}_{+}} G_{B, \mathcal{S}}$ and $E_{+}^{*}=\left(E E_{+}\right)^{*} \supset E_{+}^{*} E^{*}$. Hence $B E_{+} \subset E_{+}^{*} B$.

Note that $B E_{+}=B_{1} E_{+}+B_{2} E_{+}-B_{3} E_{+}=B_{2} E_{+}$, because $\mathcal{R}\left(E_{+}\right) \subseteq \mathcal{S}=\mathcal{N}\left(B_{1}\right)$ and $\mathcal{R}\left(E_{+}\right)=$ $\mathcal{S}_{+} \subseteq \mathcal{N}\left(B_{3}\right)$. Then

$$
B_{2} E_{+}=B E_{+} \subset E_{+}^{*} B=\left(B E_{+}\right)^{*}=\left(B_{2} E_{+}\right)^{*}=E_{+}^{*} B_{2} .
$$

Hence $E_{+}$is $B_{2}$-symmetric.

Proof of Proposition 5.11. By Lemma 5.12, $E$ admits a factorization in the form $E=E_{+}+E_{-}$, where $E_{+}$ is a $B_{2}$-symmetric semiclosed projection with range $\mathcal{S}_{+}$and $E_{-}$is a $B_{3}$-symmetric semiclosed projection with range $\mathcal{S}_{-}$.

Let us show that $P_{\mathcal{M}_{2}} B_{2}^{1 / 2}=B_{2}^{1 / 2} E_{+}$in $\mathcal{D}\left(E_{+}\right)$. In fact, if $x \in \mathcal{D}\left(E_{+}\right)$then

$$
P_{\mathcal{M}_{2}} B_{2}^{1 / 2} x=P_{\mathcal{M}_{2}} B_{2}^{1 / 2} E_{+} x+P_{\mathcal{M}_{2}} B_{2}^{1 / 2}\left(I-E_{+}\right) x .
$$

Since $E_{+} x \in \mathcal{S}_{+}, P_{\mathcal{M}_{2}} B_{2}^{1 / 2} E_{+} x=B_{2}^{1 / 2} E_{+} x$. Also, $P_{\mathcal{M}_{2}} B_{2}^{1 / 2}\left(I-E_{+}\right) x=0$ because $B_{2}^{1 / 2}\left(I-E_{+}\right) x \in$ $B_{2}^{1 / 2} \mathcal{N}\left(E_{+}\right) \subseteq B_{2}^{1 / 2}\left(B_{2}^{-1}\left(\mathcal{S}_{+}^{\perp}\right)\right)=\mathcal{R}\left(B_{2}^{1 / 2}\right) \cap B_{2}^{-1 / 2}\left(\mathcal{S}_{+}^{\perp}\right) \subseteq \mathcal{M}_{2}^{\perp}$, where we used the fact that $E_{+}$is $B_{2^{-}}$ symmetric and Proposition 4.1, Therefore, $P_{\mathcal{M}_{2}} B_{2}^{1 / 2}=B_{2}^{1 / 2} E_{+}$in $\mathcal{D}\left(E_{+}\right)$. Then, by multiplying both sides of the last equation by $B_{2}^{1 / 2}$, it follows that

$$
B_{2} E_{+} \subset B_{2}^{1 / 2} P_{\mathcal{M}_{2}} B_{2}^{1 / 2} .
$$

In a similar way, it can be proved that $P_{\mathcal{M}_{3}} B_{3}^{1 / 2}=B_{3}^{1 / 2} E_{-}$in $\mathcal{D}\left(E_{-}\right)$and $B_{3} E_{-} \subset B_{3}^{1 / 2} P_{\mathcal{M}_{3}} B_{3}^{1 / 2}$.

Therefore, if $x \in \mathcal{D}(E)=\mathcal{D}\left(E_{+}\right)=\mathcal{D}\left(E_{-}\right)$, then

$$
B E x=B E_{+} x+B E_{-} x=B_{2} E_{+} x-B_{3} E_{-} x=\left(B_{2}^{1 / 2} P_{\mathcal{M}_{2}} B_{2}^{1 / 2}-B_{3}^{1 / 2} P_{\mathcal{M}_{3}} B_{3}^{1 / 2}\right) x .
$$

Define $S:=B_{2}^{1 / 2} P_{\mathcal{M}_{2}} B_{2}^{1 / 2}-B_{3}^{1 / 2} P_{\mathcal{M}_{3}} B_{3}^{1 / 2}$, then $S \in L(\mathcal{H})^{s}$ and $B E \subset S=S^{*} \subset(B E)^{*}$. Therefore $\overline{B E}=(B E)^{*}=S$.

Let $B \in L(\mathcal{H})^{s}, \mathcal{S} \subseteq \mathcal{H}$ be a closed subspace of $\mathcal{H}$ and $E$ be a $B$-symmetric closed projection onto $\mathcal{S}$. The following example shows that if $B$ is not $\mathcal{S}$-weakly complementable then $B E$ may not admit a bounded selfadjoint extension to $\mathcal{H}$. We use the fact that a symmetric operator $T$ admits an extension to $\mathcal{H}$ if and only if $T^{*} \in L(\mathcal{H})^{s}$. Also, if $T$ is a densely defined operator such that $T \subset T^{*} \subset W \in L(\mathcal{H})$ then $W^{*}=\bar{T}=T^{*}=W$.

Example 3. As in Example 1 let $\mathcal{S} \subseteq \mathcal{H}$ be a closed subspace such that $\operatorname{dim}(\mathcal{S})=\operatorname{dim}\left(\mathcal{S}^{\perp}\right)=\infty$. Take $a \in L(\mathcal{S})^{+}$such that $\mathcal{R}(a)$ is not closed and $\overline{\mathcal{R}(a)}=\mathcal{S}$, and take $b: \mathcal{S}^{\perp} \rightarrow \mathcal{S}$ such that $b$ is invertible. Consider $B=\left[\begin{array}{cc}a & b \\ b^{*} & c\end{array}\right]$ for any $c \in L\left(\mathcal{S}^{\perp}\right)^{s}$.

Let $x:=a^{\dagger} b$ and $E=\left[\begin{array}{ll}I & x \\ 0 & 0\end{array}\right]$ where $\mathcal{D}(E)=\mathcal{S} \oplus b^{-1}(\mathcal{R}(a))$. Since $a^{\dagger}$ is closed and $b$ is bounded, $x$ is closed. Then $E$ is a closed projection onto $\mathcal{S}$.

Moreover,

$$
a x=a a^{\dagger} b=b \text { in } \mathcal{D}(x)=b^{-1}(\mathcal{R}(a))
$$

or equivalently, $a x \subset b$. Applying Proposition $4.2, E$ is $B$-symmetric.

By 25, Corollary 2], $x^{*}=\left(a^{\dagger} b\right)^{*}=b^{*} a^{\dagger}$ and then $\mathcal{D}\left(x^{*}\right)=\mathcal{D}\left(a^{\dagger}\right)=\mathcal{R}(a)$. Also, $E^{*}=\left[\begin{array}{cc}I & 0 \\ x^{*} & 0\end{array}\right]$ where $\mathcal{D}\left(E^{*}\right)=\mathcal{D}\left(x^{*}\right) \oplus \mathcal{S}^{\perp}=\mathcal{R}(a) \oplus \mathcal{S}^{\perp}$. Let us see that $\mathcal{D}\left(E^{*} B\right) \subsetneq \mathcal{H}$. In fact, let $h \in \mathcal{S} \backslash \mathcal{R}(a)$. Then $h=b k$ for some $k \in \mathcal{S}^{\perp}$, because $b$ is surjective. 
Then

$$
B k=\left[\begin{array}{cc}
a & b \\
b^{*} & c
\end{array}\right]\left[\begin{array}{l}
0 \\
k
\end{array}\right]=\left[\begin{array}{c}
h \\
c k
\end{array}\right] \notin \mathcal{D}\left(E^{*}\right) .
$$

Therefore $k \notin \mathcal{D}\left(E^{*} B\right)$ and then $\mathcal{D}\left(E^{*} B\right) \subsetneq \mathcal{H}$. Hence, by the comments above, neither $B E$ nor $E^{*} B$ admit a bounded selfadjoint extension to $\mathcal{H}$.

Proposition 5.13. Let $B \in L(\mathcal{H})^{s}$ and $\mathcal{S} \subseteq \mathcal{H}$ be a closed subspace such that $B$ is $\mathcal{S}$-weakly complementable and the matrix decomposition of $B$ is as in (5.1). If $E$ is a closed B-symmetric projection onto $\mathcal{S}$ then $E^{*} \in \mathcal{P}^{*}(B, \mathcal{S})$.

Proof. If $E$ is a closed $B$-symmetric projection onto $\mathcal{S}$ then $E^{*}$ is a densely defined closed projection with nullspace $\mathcal{S}^{\perp}$ and, by Proposition 5.11, $E^{*} B=(B E)^{*} \in L(\mathcal{H})^{s}$.

On the other hand, by Proposition [5.3 if the matrix decomposition of $E$ is as in (3.1), then $a x \subset b$. Since $B$ is $\mathcal{S}$-weakly complementable, $b=|a|^{1 / 2} f$, with $f$ the reduced solution of the equation $b=|a|^{1 / 2} h$. Then, if $a=u|a|$ is the polar decomposition of $a,\left(|a|^{1 / 2} f\right) z=\left(|a|^{1 / 2}|a|^{1 / 2} u x\right) z$ for every $z \in \mathcal{D}(x)$. Then $\left(|a|^{1 / 2} u x-f\right) \in \overline{\mathcal{R}\left(|a|^{1 / 2}\right)} \cap \mathcal{N}\left(|a|^{1 / 2}\right)=\{0\}$, so that $\left(|a|^{1 / 2} x\right) z=(u f) z$ for every $z \in \mathcal{D}(x)$. Therefore $|a|^{1 / 2} x \subset$ uf. Then $(u f)^{*} \subset\left(|a|^{1 / 2} x\right)^{*}=x^{*}|a|^{1 / 2}$ and $x^{*}|a|^{1 / 2} \in L\left(\mathcal{S}, \mathcal{S}^{\perp}\right)$. Hence $\mathcal{R}\left(|a|^{1 / 2}\right) \subseteq \mathcal{D}\left(E^{*}\right)$.

Proposition 5.14. Let $B \in L(\mathcal{H})^{s}$ and $\mathcal{S} \subseteq \mathcal{H}$ be a closed subspace such that $B \mathcal{S} \subseteq \mathcal{S}+(B \mathcal{S})^{\perp}$. Then $B$ is $\mathcal{S}$-weakly complementable and the pair $(B, \mathcal{S})$ is quasi-complementable.

Proof. Since $\mathcal{H}=\overline{B \mathcal{S}} \oplus(B \mathcal{S})^{\perp} \subseteq \overline{\mathcal{S}+(B \mathcal{S})^{\perp}}$, then $\mathcal{S}^{\perp} \cap \overline{B \mathcal{S}}=\{0\}$. By Proposition 5.2 , the pair $(B, \mathcal{S})$ is quasi-complementable, or equivalently, there exists a closed $B$-symmetric projection $E$ onto $\mathcal{S}$. If $B$ has matrix decomposition as in (5.1) and $E=\left[\begin{array}{ll}I & x \\ 0 & 0\end{array}\right]$, where $x: \mathcal{D}(x) \subseteq \mathcal{S}^{\perp} \rightarrow \mathcal{S}$ is a densely defined closed linear operator then $b^{*}=x^{*} a$, by Proposition 5.3 .

To show that $B$ is $\mathcal{S}$-weakly complementable, we estimate $\left|P_{\mathcal{S}}-P_{(B \mathcal{S})^{\perp}}\right|$. From

$$
\left(P_{\mathcal{S}}-P_{(B \mathcal{S})^{\perp}}\right)^{2}=P_{\mathcal{S}} P_{\overline{B \mathcal{S}}} P_{\mathcal{S}}+P_{\mathcal{S}^{\perp}} P_{(B \mathcal{S})^{\perp}} P_{\mathcal{S}^{\perp}},
$$

it follows that

$$
\left|P_{\mathcal{S}}-P_{(B \mathcal{S})^{\perp}}\right|=\left|P_{\overline{B \mathcal{S}}}-P_{\mathcal{S}^{\perp}}\right|=\left(P_{\mathcal{S}} P_{\overline{B \mathcal{S}}} P_{\mathcal{S}}\right)^{1 / 2}+\left(P_{\mathcal{S}^{\perp}} P_{(B \mathcal{S})^{\perp}} P_{\mathcal{S}^{\perp}}\right)^{1 / 2} .
$$

Set $\Gamma_{E^{*}}:=\left(P_{\mathcal{S}^{\perp}}+P_{\mathcal{N}^{\perp}}\right)^{1 / 2}$. Then,

$$
\mathcal{R}\left(\left(P_{\mathcal{S}} P_{\overline{B \mathcal{S}}} P_{\mathcal{S}}\right)^{1 / 2}\right) \subseteq \mathcal{R}\left(\left|P_{\overline{B \mathcal{S}}}-P_{\mathcal{S}^{\perp}}\right|\right)=\mathcal{R}\left(P_{\overline{B \mathcal{S}}}-P_{\mathcal{S}^{\perp}}\right) \subseteq \overline{B \mathcal{S}}+\mathcal{S}^{\perp} \subseteq \mathcal{N}^{\perp}+\mathcal{S}^{\perp}=\mathcal{R}\left(\Gamma_{E^{*}}\right) .
$$

On the other hand $\mathcal{R}\left(\left|P_{\mathcal{S}} B P_{\mathcal{S}}\right|\right)=\mathcal{R}\left(P_{\mathcal{S}} B P_{\mathcal{S}}\right) \subseteq \mathcal{R}\left(P_{\mathcal{S}} P_{\overline{B \mathcal{S}}} P_{\mathcal{S}}\right)$. In fact, if $y \in \mathcal{R}\left(P_{\mathcal{S}} B P_{\mathcal{S}}\right)$ then there exists $x \in \mathcal{H}$ such that $y=P_{\mathcal{S}} B P_{\mathcal{S}} x=P_{\mathcal{S}} P_{\overline{B \mathcal{S}}}\left(B P_{\mathcal{S}} x\right)$. Since $B \mathcal{S} \subseteq \mathcal{S}+(B \mathcal{S})^{\perp}$, there exists $s \in \mathcal{S}$ and $t \in(B \mathcal{S})^{\perp}$ such that $B P_{\mathcal{S}} x=s+t$. Then $y=P_{\mathcal{S}} P_{\overline{B \mathcal{S}}}(s+t)=P_{\mathcal{S}} P_{\overline{B \mathcal{S}}} P_{\mathcal{S}} s$. Therefore, by Douglas' Lemma and the monotonicity of the square root (see [36]), there exists $c \geq 0$ such that

$$
\left|P_{\mathcal{S}} B P_{\mathcal{S}}\right| \leq c^{1 / 2}\left(P_{\mathcal{S}} P_{\overline{B \mathcal{S}}} P_{\mathcal{S}}\right)
$$

or, equivalently,

$$
\mathcal{R}\left(|a|^{1 / 2}\right)=\mathcal{R}\left(\left|P_{\mathcal{S}} B P_{\mathcal{S}}\right|^{1 / 2}\right) \subseteq \mathcal{R}\left(\left(P_{\mathcal{S}} P_{\overline{B \mathcal{S}}} P_{\mathcal{S}}\right)^{1 / 2}\right) \subseteq \mathcal{R}\left(\Gamma_{E^{*}}\right)=\mathcal{D}\left(E^{*}\right)=\mathcal{D}\left(x^{*}\right) \oplus \mathcal{S}^{\perp} .
$$

Then $\mathcal{R}\left(|a|^{1 / 2}\right) \subseteq \mathcal{D}\left(x^{*}\right)$. Applying Corollary [5.8, $E^{*} B \in L(\mathcal{H})^{s}$. Finally, since $E^{*}$ is a closed densely defined projection with $\mathcal{N}\left(E^{*}\right)=\mathcal{S}^{\perp}, E^{*} B \in L(\mathcal{H})^{s}$ and $\mathcal{R}\left(|a|^{1 / 2}\right) \subseteq \mathcal{D}\left(E^{*}\right)$, by Theorem [5.9, $B$ is $\mathcal{S}$-weakly complementable.

Corollary 5.15. Let $B \in L(\mathcal{H})^{s}$ and $\mathcal{S} \subseteq \mathcal{H}$ be a closed subspace. Suppose that the matrix decomposition of $B$ is as in (5.1). If the pair $(B, \mathcal{S})$ is quasi-complementable and $\mathcal{R}\left(|a|^{1 / 2}\right) \subseteq \mathcal{R}\left(P_{\mathcal{S}} P_{\overline{B \mathcal{S}}}\right)$ then $B$ is $\mathcal{S}$-weakly complementable. 
Proof. Observe that $\mathcal{R}\left(|a|^{1 / 2}\right) \subseteq \mathcal{R}\left(P_{\mathcal{S}} P_{\overline{B \mathcal{S}}}\right)$ if and only if $\mathcal{R}\left(|a|^{1 / 2}\right) \subseteq \mathcal{R}\left(\left(P_{\mathcal{S}} P_{\overline{B \mathcal{S}}} P_{\mathcal{S}}\right)^{1 / 2}\right)$. Let $E=P_{\mathcal{S} / / \mathcal{N}}$, with $\mathcal{N} \subseteq(B \mathcal{S})^{\perp}$. Then, if $\mathcal{R}\left(|a|^{1 / 2}\right) \subseteq \mathcal{R}\left(\left(P_{\mathcal{S}} P_{\overline{B S}} P_{\mathcal{S}}\right)^{1 / 2}\right)$, by using arguments similar to those found in the proof of Proposition 5.14 we get that $E^{*}$ is a closed densely defined projection with $\mathcal{N}\left(E^{*}\right)=\mathcal{S}^{\perp}$, $E^{*} B \in L(\mathcal{H})^{s}$ and $\mathcal{R}\left(|a|^{1 / 2}\right) \subseteq \mathcal{D}\left(E^{*}\right)$. Then, by Theorem [5.9, $B$ is $\mathcal{S}$-weakly complementable.

\subsection{Applications: Schur complements of selfadjoint operators}

We recall the definition of Schur complement for an $\mathcal{S}$-weakly complementable selfadjoint operator.

Definition. Let $B \in L(\mathcal{H})^{s}$ and $\mathcal{S} \subseteq \mathcal{H}$ be a closed subspace such that $B$ is $\mathcal{S}$-weakly complementable. When $B$ is as in (5.1), let $f$ be the reduced solution of $b=|a|^{1 / 2} x$ and $a=u|a|$ the polar decomposition of $a$. The Schur complement of $B$ to $\mathcal{S}$ is defined as

$$
B / \mathcal{S}:=\left[\begin{array}{cc}
0 & 0 \\
0 & c-f^{*} u f
\end{array}\right]
$$

and $B_{\mathcal{S}}:=B-B / \mathcal{S}$ is the compression of $B$ to $\mathcal{S}$.

When $B \in L(\mathcal{H})^{+}$this formula gives the usual Schur complement, see [1, Theorem 3].

When the operator $B$ is $\mathcal{S}$-complementable, the Schur complement can be written as $B_{/ \mathcal{S}}=(I-F) B$, for any bounded projection with $\mathcal{N}(F)=\mathcal{S}^{\perp}$ such that $(F B)^{*}=F B$. In fact, from [14, Corollary 3.12] it suffices to take $F=Q^{*}$, for any $Q \in \mathcal{P}(B, \mathcal{S})$.

A similar formula for $B / \mathcal{S}$ can be given when $B$ is $\mathcal{S}$-weakly complementable. In this case the projection need not be bounded, but it is a semiclosed densely defined projection with closed nullspace.

Theorem 5.16 (cf. [14, Theorem 3.14]). Let $B \in L(\mathcal{H})^{s}$ and $\mathcal{S}$ be a closed subspace of $\mathcal{H}$. Suppose that $B$ is $\mathcal{S}$-weakly complementable then

$$
B / \mathcal{S}=(I-E) B
$$

for every $E \in \mathcal{P}^{*}(B, \mathcal{S})$.

Proof. Let $E \in \mathcal{P}^{*}(B, \mathcal{S})$. Suppose that the matrix decomposition of $E$ is as in (5.6) and that of $B$ is as in (5.1). Then, by Lemma 5.6. $y a=b^{*}$ and since $\mathcal{R}\left(|a|^{1 / 2}\right) \subseteq \mathcal{D}(E)$, by Corollary 2.7, $E|a|^{1 / 2} \in L(\mathcal{S}, \mathcal{H})$, so that $y|a|^{1 / 2} \in L\left(\mathcal{S}, \mathcal{S}^{\perp}\right)$. Hence

$$
(I-E) B=\left[\begin{array}{cc}
0 & 0 \\
-y & I
\end{array}\right]\left[\begin{array}{cc}
a & b \\
b^{*} & c
\end{array}\right]=\left[\begin{array}{cc}
0 & 0 \\
-y a+b^{*} & c-y b
\end{array}\right]=\left[\begin{array}{cc}
0 & 0 \\
0 & c-y b
\end{array}\right] .
$$

Let $f$ be the reduced solution of $b=|a|^{1 / 2} x$ and $a=u|a|$ the polar decomposition of $a$. Then $y b=f^{*} u f$. In fact, since $y a=b^{*}$ we have that $y|a|=f^{*}|a|^{1 / 2} u=f^{*} u|a|^{1 / 2}$. Then $y|a|^{1 / 2}=f^{*} u$ on $\mathcal{R}\left(|a|^{1 / 2}\right)$, and since $y|a|^{1 / 2}$ is bounded, $y|a|^{1 / 2}=f^{*} u$ on $\overline{\mathcal{R}\left(|a|^{1 / 2}\right)}$. Then $y b=y|a|^{1 / 2} f=f^{*} u f$ because $\mathcal{R}(f) \subseteq \overline{\mathcal{R}\left(|a|^{1 / 2}\right)}$. Hence $(I-E) B=B / \mathcal{S}$.

In particular, Theorem 5.16 gives a formula for the Schur complement of any positive operator $B$ to $\mathcal{S}$ in terms of semiclosed projections.

Different definitions where given for the minus order, for example, using generalized inverses in the matrix case, see [33]. We give the following definition, equivalent to those appearing in [38] and [20].

Definition. Let $A, B \in L(\mathcal{H})$, we write $A \overline{\leq} B$ if there exist projections $P, Q \in \mathcal{Q}$ such that $A=P B$ and $A^{*}=Q B^{*}$.

It was proved in [38] and [20] that $\overline{\leq}$ is a partial order, known as the minus order for operators. In [6], it was shown that $A \overline{\leq} B$ if and only if the sets $\overline{\mathcal{R}(A)} \dot{+} \overline{\mathcal{R}(B-A)}$ and $\overline{\mathcal{R}\left(A^{*}\right)}+\overline{\mathcal{R}\left(B^{*}-A^{*}\right)}$ are closed. In 
20, Theorem 3.3], another characterization of the minus order in terms of the range additivity property was given:

$$
A \overline{\leq} B \text { if and only if } \mathcal{R}(B)=\mathcal{R}(A) \dot{+} \mathcal{R}(B-A) \text { and } \mathcal{R}\left(B^{*}\right)=\mathcal{R}\left(A^{*}\right) \dot{+} \mathcal{R}\left(B^{*}-A^{*}\right) .
$$

In view of this equivalence, the left minus order was defined in [20] for operators in $L(\mathcal{H})$. This notion is weaker than the minus order, in the infinite dimensional setting.

Definition. Let $A, B \in L(\mathcal{H})$, we write $A_{-} \leq B$ if $\mathcal{R}(B)=\mathcal{R}(A) \dot{+} \mathcal{R}(B-A)$.

The relation ${ }_{-} \leq$is a partial order, see [20]. The following is a characterization of the left minus order in terms of a semiclosed projection.

Proposition 5.17 (cf. [20, Proposition 3.13]). Let $A, B \in L(\mathcal{H})$. Then $A_{-} \leq B$ if and only if there exists $P \in \mathcal{Q}_{S C}$ such that $A=P B$ and $\mathcal{R}(A) \subseteq \mathcal{R}(B)$.

Proof. Suppose that $A_{-} \leq B$ then $\mathcal{R}(B)=\mathcal{R}(A) \dot{+} \mathcal{R}(B-A)$ so that $\mathcal{R}(A) \subseteq \mathcal{R}(B)$. Define $P=$ $P_{\mathcal{R}(A) / / R(B-A) \oplus \mathcal{N}\left(B^{*}\right)}$. Then $P \in \mathcal{Q}_{S C}$ and it is easy to check that $A=P B$

Conversely, suppose that $A=P B$ for $P \in \mathcal{Q}_{S C}$ and $\mathcal{R}(A) \subseteq \mathcal{R}(B)$. Then $\mathcal{R}(B)=\mathcal{R}(A)+\mathcal{R}(B-A)$ and the sum is direct because $\mathcal{R}(A) \subseteq \mathcal{R}(P)$ and $\mathcal{R}(B-A) \subseteq \mathcal{N}(P)$.

A different generalization of the minus order was introduced by Arias et al. in [8]:

Definition. Given $A, B \in L(\mathcal{H})$, we write $A \prec B$ if there exist $Q, P \in \mathcal{Q}_{S C}$ with closed ranges such that $A=Q B$ and $A^{*}=P B^{*}$.

The relation $\prec$ is a partial order in $L(\mathcal{H})$ (see [8, Lemma 4.5]). In [ㅇ, Lemma 4.4], it was proved that $A \prec B$ if and only if $\overline{\mathcal{R}(A)} \cap \mathcal{R}(B-A)=\{0\}$ and $\overline{\mathcal{R}\left(A^{*}\right)} \cap \mathcal{R}\left(B^{*}-A^{*}\right)=\{0\}$.

Let $B \in L(\mathcal{H})^{s}$ and $\mathcal{S}$ be a closed subspace of $\mathcal{H}$. Suppose that the matrix decomposition of $B$ is as in (5.1). Denote by $\tilde{\mathcal{Q}}:=\left\{Q \in \mathcal{Q}_{S C}: \mathcal{R}(Q)\right.$ is closed and $\left.\mathcal{R}\left(|a|^{1 / 2}\right) \oplus \mathcal{S}^{\perp} \subseteq \mathcal{D}(Q)\right\}$. Note that if $E \in \mathcal{P}^{*}(B, \mathcal{S})$ then $I-E \in \tilde{Q}$. Define

$$
\mathcal{M}(B, \mathcal{S}):=\left\{X \in L(\mathcal{H})^{s}: \mathcal{R}(X) \subseteq \mathcal{S}^{\perp} \text { and } X=Q B, \text { for some } Q \in \tilde{Q}\right\} .
$$

Theorem 5.18. Let $B \in L(\mathcal{H})^{s}$ and $\mathcal{S}$ be a closed subspace of $\mathcal{H}$ such that $B$ is $\mathcal{S}$-weakly complementable. Then

$$
B / \mathcal{S}=\max _{\prec} \mathcal{M}(B, \mathcal{S}) .
$$

Proof. Let $E \in \mathcal{P}^{*}(B, \mathcal{S})$. Then, by Theorem [5.16, $B / \mathcal{S}=(I-E) B \in L(\mathcal{H})^{s}$ and $\mathcal{R}(B / \mathcal{S}) \subseteq \mathcal{S}^{\perp}$. Set $Q:=I-E$, then $Q \in \tilde{Q}$ and $B / \mathcal{S}=Q B$. So that $B / \mathcal{S} \in \mathcal{M}(B, \mathcal{S})$.

On the other hand, let $X \in \mathcal{M}(B, \mathcal{S})$. Then $X \in L(\mathcal{H})^{s}, \mathcal{R}(X) \subseteq \mathcal{S}^{\perp}$ and there exists $Q \in \tilde{Q}$ such that $X=Q B$. Suppose that the matrix decomposition of $B$ is as in (5.1) and let $f$ be the reduced solution of $b=|a|^{1 / 2} x$ and $a=u|a|$ the polar decomposition of $a$. Then

$$
B=\left[\begin{array}{cc}
a & |a|^{1 / 2} f \\
f^{*}|a|^{1 / 2} & c
\end{array}\right]=\Gamma U \Gamma,
$$

where $\Gamma:=\left[\begin{array}{cc}|a|^{1 / 2} & 0 \\ 0 & I\end{array}\right] \in L(\mathcal{H})^{+}$and $U:=\left[\begin{array}{cc}u & f \\ f^{*} & c\end{array}\right] \in L(\mathcal{H})^{s}$.

Since $Q$ is semiclosed and $\mathcal{R}(\Gamma)=\mathcal{R}\left(|a|^{1 / 2}\right) \oplus \mathcal{S}^{\perp} \subseteq \mathcal{D}(Q)$, by Corollary 2.7 $Q \Gamma \in L(\mathcal{H})$. Therefore

$$
X=Q B=Q \Gamma U \Gamma=X^{*}=(Q \Gamma U \Gamma)^{*}=\Gamma U(Q \Gamma)^{*} .
$$

Let $E \in \mathcal{P}^{*}(B, \mathcal{S})$. Since $\mathcal{R}(\Gamma) \subseteq \mathcal{D}(E)=\mathcal{D}(I-E)$, by Corollary 2.7. $(I-E) \Gamma \in L(\mathcal{H})$. Also,

$$
\begin{aligned}
X & =(I-E) X=(I-E) \Gamma U(Q \Gamma)^{*}=X^{*}=\left((I-E) \Gamma U(Q \Gamma)^{*}\right)^{*} \\
& =Q \Gamma U((I-E) \Gamma)^{*}=Q((I-E) \Gamma U \Gamma)^{*}=Q((I-E) B)^{*}=Q B / \mathcal{S},
\end{aligned}
$$

where we used Theorem 5.16 Then $X \prec B / \mathcal{S}$. 


\section{Acknowledgements}

We thank the anonymous referee for carefully reading our manuscript and helping us to improve it with several useful comments.

Maximiliano Contino was supported by the UBA's Strategic Research Fund 2018 and CONICET PIP 0168. Alejandra Maestripieri was supported by CONICET PIP 0168. The work of Stefania Marcantognini was done during her stay at the Instituto Argentino de Matemática with an appointment funded by the CONICET. She is greatly grateful to the institute for its hospitality and to the CONICET for financing her post.

\section{References}

[1] Anderson W.N., Trapp G.E., Shorted Operators II, SIAM J. Appl. Math., 28 (1975), 60-71.

[2] Ando T., Generalized Schur complements, Linear Algebra Appl., 27 (1979), 173-186.

[3] Ando T., De Branges spaces and analytic operator functions, Lecture Notes, Hokkaido University, Sapporo, Japan (1990).

[4] Ando T., Unbounded or bounded idempotent operators in Hilbert spaces, Linear Algebra Appl., 438 (2013), 3769-3775.

[5] Ando T., Bunce J., Trapp G., An alternative variational characterization of matrix Riccati equation solutions, Circ. Syst. Signal Pr., 9 (1990), 223-228.

[6] Antezana J., Corach G., Stojanoff D., Bilateral shorted operators and parallel sums, Linear Algebra Appl., 414 (2006), 570-588.

[7] Arias M.L., Corach G., Gonzalez M.C., Products of projections and positive operators, Linear Algebra Appl., 439 (2013), 1730-1741.

[8] Arias M.L., Corach G., Maestripieri A., On complementable operators in the sense of T. Ando, Linear Algebra Appl. 594 (2020), 158-176.

[9] Arias M.L., Corach G., Maestripieri A., Range additivity, shorted operator and the ShermanMorrison-Woodbury formula, Linear Algebra Appl., 467 (2015), 86-99.

[10] Balaji S., Johnson S.P., On semiclosed subspaces of Hilbert spaces, Int. J. Pure Appl. Math., 79 (2012), 249-258.

[11] Ball J.A., Bolotnikov V., De Branges-Rovnyak spaces: basics and theory, Operator Theory, (2015), $1-45$.

[12] Caradus S.R., Semiclosed operators, Pac. J. Math., 44 (1973), 75-79.

[13] Carlson D., Haynsworth E.V., Complementable and almost definite matrices, Linear Algebra Appl., 52 (1983), 157-176.

[14] Contino M., Maestripieri A., Marcantognini S., Schur complements of selfadjoint Krein space operators, Linear Algebra Appl., 581 (2019), 214-246.

[15] Corach G., Gonzalez M. C., Maestripieri A., Unbounded symmetrizable idempotens, Linear Algebra Appl., 437 (2012), 659-674.

[16] Corach G., Maestripieri A., Products of orthogonal projections and polar decompositions, Linear Algebra Appl., 434 (2011), 1594-1609.

[17] Corach G., Maestripieri A., Stojanoff D., Oblique projections and Schur complements, Acta Sci. Math. (Szeged), 67 (2001), 337-356. 
[18] de Branges L., Rovnyak J., Square Summable Power Series, Holt, Rinehart and Winston (1966).

[19] de Branges L., Rovnyak J., Canonical models in quantum scattering theory, in Perturbation Theory and its Applications in Quantum Mechanics, ed. by C.H. Wilcox, Wiley (1966), 295-392.

[20] Djikić M.S., Fongi G., Maestripieri A., The minus order and range additivity, Linear Algebra Appl., 531 (2017), 234-256.

[21] Djikić M.S., Radenković J.N., Simultaneous extension of two bounded operators between Hilbert spaces, J. Operator Theory, 83 (2020), 229-250.

[22] Douglas R.G., On majorization, factorization and range inclusion of operators in Hilbert space, Proc. Amer. Math. Soc., 17 (1966), 413-416.

[23] Fillmore P.A.,Williams J.P., On operator ranges, Advances in Math., 7 (1971), 254-281.

[24] Foiaş C., Invariant Para-closed Subspaces, Indiana U. Math. J., 10 (1972), 887-906.

[25] Holland S. S., On the Adjoint of the Product of Operators, J. Funct. Anal., 3 (1969), 337-344.

[26] Izumino S.,Quotients of bounded operators, Proc. Amer. Math. Soc., 106 (1989), 427-435.

[27] Izumino S.,Quotients of bounded operators and their weak adjoints, J. Operator Theory, 29 (1993), 83-96.

[28] Izumino S., The product of operators with closed range and an extension of the reverse order law, Tohoku Math. J., 34 (1982), 43-52.

[29] Kaufman W.E., Semiclosed operators in Hilbert space, Proc. Amer. Math. Soc., 76 (1979), 67-73.

[30] Koliha J.J., On Kaufman's theorem, J. Math. Anal. Appl., 411 (2014), no.2, 688-692.

[31] Mac Nearney J.S., Investigation concerning positive definite continued fractions, Duke Math. J., 26 (1959), 663-678.

[32] Mac Nearney J.S., Continuous embeddings of Hilbert spaces, Rend. Circ. Mat. Palmero, 19 (1970), 109-112.

[33] Mitra S.K., The minus partial order and the shorted matrix, Linear Algebra Appl., 83 (1986), 1-27.

[34] Nashed M.Z., Inner, outer and generalized inverses in Banach and Hilbert spaces, Numer. Funct. Anal. Optim., 9 (1987), 261-325.

[35] Ôta S., Unbounded nilpotents and idempotents, J. Math. Anal. Appl., 132 (1988), 300-398.

[36] Pedersen G.K., Some operator monotone functions, Proc. Amer. Math. Soc., 36 (1972), 309-310.

[37] Sebestyén Z., Restrictions of positive operators, Acta Sci. Math., 46 (1983), 299-301.

[38] Šemrl P., Automorphisms of $B(\mathcal{H})$ with respect to minus partial order, J. Math. Anal. Appl., 369 (2010), 205-213. 